\title{
All-inclusive interacting dark sector cosmologies
}

\author{
Weiqiang Yang $\odot,{ }^{1, *}$ Eleonora Di Valentino, ${ }^{2, \uparrow}$ Olga Mena, ${ }^{3, *}$ Supriya Pan, ${ }^{4, \S}$ and Rafael C. Nunes ${ }^{5, \|}$ \\ ${ }^{1}$ Department of Physics, Liaoning Normal University, Dalian, 116029, People's Republic of China \\ ${ }^{2}$ Jodrell Bank Center for Astrophysics, School of Physics and Astronomy, University of Manchester, \\ Oxford Road, Manchester, M13 9PL, United Kingdom \\ ${ }^{3}$ IFIC, Universidad de Valencia-CSIC, 46071, Valencia, Spain \\ ${ }^{4}$ Department of Mathematics, Presidency University, 86/1 College Street, Kolkata 700073, India \\ ${ }^{5}$ Divisão de Astrofísica, Instituto Nacional de Pesquisas Espaciais, Avenida dos Astronautas 1758, \\ São José dos Campos, 12227-010, SP, Brazil
}

(Received 9 February 2020; accepted 24 March 2020; published 6 April 2020)

\begin{abstract}
In this paper we explore possible extensions of interacting dark energy cosmologies, where dark energy and dark matter interact nongravitationally with one another. In particular, we focus on the neutrino sector, analyzing the effect of both neutrino masses and the effective number of neutrino species. We consider the Planck 2018 legacy release data combined with several other cosmological probes, finding no evidence for new physics in the dark radiation sector. The current neutrino constraints from cosmology should therefore be regarded as robust, as they are not strongly dependent on the dark sector physics, once all the available observations are combined. Namely, we find a total neutrino mass $M_{\nu}<0.15 \mathrm{eV}$ and a number of effective relativistic degrees of freedom $N_{\text {eff }}=3.03_{-0.33}^{+0.33}$, both at $95 \%$ C.L., which are close to those obtained within the $\Lambda$ CDM cosmology, $M_{\nu}<0.12 \mathrm{eV}$ and $N_{\text {eff }}=3.00_{-0.35}^{+0.36}$, for the same data combination.
\end{abstract}

DOI: 10.1103/PhysRevD.101.083509

\section{INTRODUCTION}

An overwhelming number of observations confirm that our Universe is undergoing a phase of accelerated expansion. Within the context of general relativity, this effect can be described by adding a dark energy (DE) component, characterized by a negative pressure. According to the observational evidence, nearly $68 \%$ of the total energy budget of the Universe consists of such a dark energy fluid. Observations further predict that around 28\% of the total energy budget of the Universe corresponds to nonluminous dark matter (DM) [1-3]. Observations from different astronomical sources seem to point to a DE component that is very similar to a cosmological constant, and to a pressureless DM component, also known as cold dark matter (CDM). These two components are the basic ingredients of the socalled $\Lambda \mathrm{CDM}$ cosmology. However, given the fact that (a) the precise nature of the DE and DM fluids remains unknown, despite the large number of devoted measurements to unravel the underlying physics [4-8], and (b) due to a number of persisting tensions within the minimal $\Lambda \mathrm{CDM}$ scheme, there is still plenty of room for other possible scenarios with nonminimal dark sector physics. In this

\footnotetext{
d11102004@163.com

eleonora.divalentino@manchester.ac.uk

omena@ific.uv.es

\$supriya.maths@presiuniv.ac.in

"rafadcnunes@gmail.com
}

regard, we shall consider here interacting dark sector cosmologies, in which the DE and the DM components interact nongravitationally [9-45]. Indeed, the dynamics of such a universe could have been present since early times, modifying other dark sector physics, such as that of dark radiation, i.e., that of the neutrinos. Therefore, the question we would like to address here is the following: are cosmological limits on the standard physics of dark radiation strongly dependent on the dark sector model?

In order to investigate this issue we use a set of observational data from various important cosmological sources. This set includes the cosmic microwave background radiation, baryon acoustic oscillation distance measurements, local measurements of the Hubble constant from the Hubble Space Telescope, the Pantheon sample of type Ia supernovae, and finally the Hubble parameter measurements at different redshifts from cosmic chronometers.

The structure of the paper is as follows. Section II introduces the cosmological model explored here, while Sec. III describes the methodology and measurements exploited in our data analyses. Section IV presents our results, and we conclude the paper in Sec. V.

\section{INTERACTING DARK UNIVERSE}

We consider an interacting dark sector scenario between cold DM and DE in a homogeneous and isotropic flat universe where the gravitational sector is described by Einstein's general relativity. The conservation of the 
energy-momentum tensor for cold DM and DE leads to the coupled equations

$$
\begin{gathered}
\dot{\rho}_{c}+3 H \rho_{c}=-Q, \\
\dot{\rho}_{x}+3 H\left(1+w_{x}\right) \rho_{x}=Q,
\end{gathered}
$$

where we have explicitly used the DE state parameter $w_{x}=$ $p_{x} / \rho_{x}$ (here $p_{x}$ is the pressure of the DE fluid and $\rho_{x}$ is its energy density), while for DM we used its pressure $p_{c}$ (which for a CDM component is 0 ) and energy density $\rho_{c}$. Note that here $H$ denotes the Hubble rate of the prescribed homogeneous and isotropic universe, i.e., a Friedmann-LemaîtreRobertson-Walker universe. In Eq. (1) we have introduced the quantity $Q$, which is known as the interaction rate between the dark sectors. While the exact form of the interaction rate is not known, possible phenomenological descriptions at the classical and quantum levels have been developed in the literature (see Ref. [46] for a comprehensive review) in an attempt to recover some different interaction models from scalar field theories; see, e.g., the recent Ref. [47], or for other cosmological contexts see, e.g., Ref. [48]. In the present work, we restrict ourselves to the most well-known parametric form of the coupling function $Q$ :

$$
Q=3 H \xi \rho_{x},
$$

where $\xi$ is the coupling parameter that characterizes the strength of the coupling. For convenience, the condition $\xi<0$ corresponds to energy flow from $\mathrm{DE}$ to $\mathrm{DM}$, and $\xi>0$ represents the opposite case. We refer to Refs. $[27,31,34,40,41]$ for details concerning the linear perturbation theory within these coupled cosmologies.

\section{OBSERVATIONAL DATA AND METHODOLOGY}

We now briefly describe the observational data sets that we use in the present work.

(1) Cosmic microwave background (CMB): We make use of the latest $\mathrm{CMB}$ measurements from the final 2018 Planck legacy release [1-3].

(2) Baryon acoustic oscillations (BAO): A number of BAO constraints from different astronomical missions are exploited, namely, those from the 6dFGS [49], SDSS-MGS [50], and BOSS DR12 [51] surveys, as considered by the Planck Collaboration [2].

(3) R19: We adopt the latest measurement of the Hubble constant obtained by a reanalysis of the Hubble Space Telescope data using Cepheids as calibrators, i.e., $H_{0}=74.03 \pm 1.42 \mathrm{~km} / \mathrm{s} / \mathrm{Mpc}$ at $68 \%$ C.L. [52].

(4) Pantheon: We analyze the luminosity distance data of type Ia supernovae from the Pantheon catalog [53], including 1048 data points in the redshift region $z \in[0.01,2.3]$.

(5) Cosmic chronometers (CC): Thirty measurements of the Hubble parameter at different redshifts extracted from cosmic chronometers in the redshift range $0<z<2$ (as tabulated in Ref. [54]) are also considered in our data analyses.

We consider a fiducial cosmology described by eight cosmological parameters: six from the standard $\Lambda \mathrm{CDM}$ model (the baryon and cold dark matter energy densities $\Omega_{\mathrm{b}} h^{2}$ and $\Omega_{\mathrm{c}} h^{2}$, the ratio between the sound horizon and angular diameter distance at decoupling $100 \theta_{M C}$, the reionization optical depth $\tau$, and the spectral index and amplitude of the scalar primordial power spectrum $n_{s}$ and $A_{s}$ ), and two that account for the dark sector physics (the dark energy equation of state $w_{x}$ and the strength of the coupling $\xi$ ). Therefore, the interacting dark energy (IDE) scenario is described by

$\mathcal{P} \equiv\left\{\Omega_{b} h^{2}, \Omega_{c} h^{2}, 100 \theta_{M C}, \tau, n_{s}, \log \left[10^{10} A_{s}\right], \xi, w_{x}\right\}$.

We consider $\xi<0$ in the phantom scenario $\left(w_{x}<-1\right)$ and $\xi>0$ in the quintessence regime $\left(w_{x}>-1\right)$ to avoid early-time instabilities $[13,14]$; see Table I for the priors on all of the parameters. It is important to mention that the division of the parameter space into $\left(\xi<0, w_{x}<-1\right)$ or $\left(\xi>0, w_{x}>-1\right)$ is motivated by the doom factor analysis [14]. Following the notation of Refs. [26,27,40], the doom factor $d$ is defined as $d=-Q\left[3 H\left(1+w_{x}\right) \rho_{x}\right]^{-1}$. The interacting model becomes stable for $d<0$, which for the present model $Q=3 H \xi \rho_{x}$ requires either $\xi<0, w_{x}<$ -1 or $\xi>0, w_{x}>-1$. Hence, as we can see this division is necessary to maintain the early-time instability in the perturbation evolution of the scalar modes of the dark species (DM and DE). In this paper, we refer to the scenario with a phantom-like DE equation of state $\left(w_{x}<-1\right)$ as IDEp, and the IDE scenario with a quintessence-like DE equation of state $\left(w_{x}>-1\right)$ as IDEq.

Then, we allow for freedom in the dark radiation sector by enlarging the fiducial cosmological scenario with the sum of the neutrino masses $M_{\nu}\left(\mathrm{IDE}+M_{\nu}\right)$,

$\mathcal{P} \equiv\left\{\Omega_{b} h^{2}, \Omega_{c} h^{2}, 100 \theta_{M C}, \tau, n_{s}, \log \left[10^{10} A_{s}\right], \xi, w_{x}, M_{\nu}\right\}$,

TABLE I. List of the flat priors on the cosmological parameters assumed in this work.

\begin{tabular}{lcc}
\hline \hline Parameter & Prior phantom & Prior quintessence \\
\hline$\Omega_{\mathrm{b}} h^{2}$ & {$[0.013,0.033]$} & {$[0.013,0.033]$} \\
$\Omega_{\mathrm{c}} h^{2}$ & {$[0.001,0.99]$} & {$[0.001,0.99]$} \\
$100 \theta_{M C}$ & {$[0.5,10]$} & {$[0.5,10]$} \\
$\tau$ & {$[0.01,0.8]$} & {$[0.01,0.8]$} \\
$n_{\mathrm{S}}$ & {$[0.7,1.3]$} & {$[0.7,1.3]$} \\
$\log \left[10^{10} A_{s}\right]$ & {$[1.7,5.0]$} & {$[1.7,5.0]$} \\
$w_{x}$ & {$[-3,-1]$} & {$[-1,0]$} \\
$\xi$ & {$[-1,0]$} & {$[0,1]$} \\
$M_{\nu}$ & {$[0,1]$} & {$[0,1]$} \\
$N_{\text {eff }}$ & {$[0.05,10]$} & {$[0.05,10]$} \\
\hline \hline
\end{tabular}


or with extra-relativistic species at recombination $\left(\mathrm{IDE}+N_{\text {eff }}\right)$,

$\mathcal{P} \equiv\left\{\Omega_{b} h^{2}, \Omega_{c} h^{2}, 100 \theta_{M C}, \tau, n_{s}, \log \left[10^{10} A_{s}\right], \xi, w_{x}, N_{\text {eff }}\right\}$

Finally, we also analyze the full scenario $\mathrm{IDE}+M_{\nu}+N_{\mathrm{eff}}$ :

$$
\begin{aligned}
\mathcal{P} \equiv & \left\{\Omega_{b} h^{2}, \Omega_{c} h^{2}, 100 \theta_{M C}, \tau, n_{s}, \log \left[10^{10} A_{s}\right],\right. \\
& \left.\xi, w_{x}, M_{\nu}, N_{\text {eff }}\right\} .
\end{aligned}
$$

For numerical purposes, we make use of the latest version of the publicly available Markov chain Monte Carlo code COSMOMC [55,56] package which supports the new 2018 Planck likelihood [1] and has been modified to include IDE scenarios. The convergence diagnostic follows the GelmanRubin criteria [57].

\section{RESULTS}

Throughout this section we present the results obtained within the different IDE scenarios.

\section{A. IDE with $w_{x}<-1$}

Here we start by analyzing the constraints obtained under the assumption of a phantom-like dark energy equation of state $w_{x}<-1$.

\section{IDE}

The results for our baseline IDE scenario, based on eight parameters, with a dark energy equation of state $w_{x}<-1$ are shown in Table II and Fig. 1.

Notice that, regardless of the data set combination, the CDM energy density $\Omega_{c} h^{2}$ is larger than that in the $\Lambda \mathrm{CDM}$ model. This is mainly due to the energy flow between the dark sectors which, for this phantom case with $\xi<0$ (i.e., energy flux flowing from DE to DM), results in a larger density for cold dark matter at present; see also Ref. [58]. Also, the Hubble constant is always much larger than that in the canonical $\Lambda \mathrm{CDM}$ scenario when considering $\mathrm{CMB}$ only, due to the fact that in the phantom region there is a strong degeneracy between $w_{x}$ and $H_{0}$ at the level of the CMB (see Fig. 1). When the dark energy equation of state is allowed to vary in the $w<-1$ region, $H_{0}$ must be larger to prevent a shift in the CMB peaks. Notice that here there is no preference for a nonzero dark sector coupling, and the socalled Hubble constant tension is greatly alleviated due to the phantom character of the DE component and not from the presence of a coupling. From CMB measurements alone, we find that a dark energy equation of state $w_{x}<-1$ is preferred at a significance above the $2 \sigma$ level and a lower limit of $\xi>-0.090$ at $95 \%$ C.L. The value of $S_{8}$ is instead shifted in the right direction to solve the tension between Planck and the cosmic shear experiments DES [59,60], KiDS-450 [61-63], or CFHTLenS [64-66], i.e., $S_{8}=$ $0.756 \pm 0.034$ at $68 \%$ C.L. for CMB in the IDE model, to be compared with $S_{8}=0.822 \pm 0.015$ at $68 \%$ C.L. in the $\Lambda \mathrm{CDM}$ scenario for the same data set, or $S_{8}=0.777_{-0.036}^{+0.022}$ at $68 \%$ C.L. for a $w$ CDM cosmology. When including the BAO data (see the third column of Table II), the six parameters of the standard $\Lambda \mathrm{CDM}$ model are almost unmodified, while $w_{x}$ is now perfectly consistent with a cosmological constant at the $2 \sigma$ level. For $\mathrm{CMB}+\mathrm{BAO}, H_{0}$ shifts back towards a lower value $\left(H_{0}=68.7_{-25}^{+2.7} \mathrm{~km} / \mathrm{s} / \mathrm{Mpc}\right.$ at $95 \%$ C.L. $)$. The value of the clustering parameter $\sigma_{8}$ is still below those in $\Lambda \mathrm{CDM}$ and $w \mathrm{CDM}$, due to the correlation with $\xi$ (see Fig. 1). When considering the combination of CMB + R19 (shown in the fourth column of Table II), we find a value for the Hubble constant in agreement with the R19 measurement, together with a preference at more than $3 \sigma$ for a phantom

TABLE II. $\quad 95 \%$ C.L. constraints within the minimal IDE model in the phantom-like dark energy scenario $\left(w_{x}<-1\right)$ from several data set combinations, where $\mathrm{CB}, \mathrm{CR} 19, \mathrm{CPCC}, \mathrm{CBPCC}$, and CR19PCC correspond to the combinations CMB $+\mathrm{BAO}$, CMB $+\mathrm{R} 19$,

\begin{tabular}{|c|c|c|c|c|c|c|}
\hline Parameters & $\mathrm{CMB}$ & $\mathrm{CB}$ & CR19 & СРCC & CBPCC & CR19PCC \\
\hline$\overline{\Omega_{c} h^{2}}$ & $0.134_{-0.015}^{+0.017}$ & $0.135_{-0.014}^{+0.014}$ & $0.134_{-0.015}^{+0.017}$ & $0.135_{-0.014}^{+0.015}$ & $0.135_{-0.014}^{+0.014}$ & $0.134_{-0.015}^{+0.016}$ \\
\hline$\Omega_{b} h^{2}$ & $0.02239_{-0.00030}^{+0.00030}$ & $0.02239_{-0.00028}^{+0.00014+0.00029}$ & $0.02238_{-0.00029}^{+0.00029}$ & $0.02236_{-0.00030}^{+0.00030}$ & $0.02240_{-0.00028}^{+0.00028}$ & $0.02242_{-0.00029}^{+0.00028}$ \\
\hline $100 \theta_{M C}$ & $1.0402_{-0.0011}^{+0.0011}$ & $1.0401_{-0.0010}^{+0.0010}$ & $1.0402_{-0.0011}^{+0.0010}$ & $1.0401_{-0.0010}^{+0.0010}$ & $1.0401_{-0.0010}^{+0.0010}$ & $1.0402_{-0.0010}^{+0.0010}$ \\
\hline$\tau$ & $0.054_{-0.015}^{+0.015}$ & $0.055_{-0.015}^{+0.016}$ & $0.054_{-0.015}^{+0.016}$ & $0.054_{-0.015}^{+0.016}$ & $0.055_{-0.015}^{+0.016}$ & $0.055_{-0.015}^{+0.016}$ \\
\hline$n_{s}$ & $0.9652_{-0.0086}^{+0.0086}$ & $0.9657_{-0.0082}^{+0.00081}$ & $0.9648_{-0.0086}^{+0.0086}$ & $0.9647_{-0.0086}^{+0.0086}$ & $0.9660_{-0.0080}^{+0.0078}$ & $0.9662_{-0.0085}^{+0.0085}$ \\
\hline $\ln \left(10^{10} A_{s}\right)$ & $3.043_{-0.031}^{+0.029}$ & $3.045_{-0.030}^{+0.032}$ & $3.044_{-0.030}^{+0.031}$ & $3.045_{-0.031}^{+0.033}$ & $3.045_{-0.031}^{+0.033}$ & $3.045_{-0.031}^{+0.033}$ \\
\hline$w_{x}$ & $-1.58_{-0.44}^{+0.49}$ & $-1.094_{-0.099}^{+0.094}$ & $-1.27_{-0.12}^{+0.11}$ & $-1.085_{-0.078}^{+0.083}$ & $-1.080_{-0.072}^{+0.078}$ & $-1.139_{-0.083}^{+0.081}$ \\
\hline$\xi$ & $>-0.090$ & $>-0.101$ & $>-0.094$ & $>-0.100$ & $>-0.101$ & $>-0.099$ \\
\hline$\Omega_{m 0}$ & $0.23_{-0.09}^{+0.11}$ & $0.336_{-0.042}^{+0.044}$ & $0.286_{-0.035}^{+0.038}$ & $0.338_{-0.038}^{+0.040}$ & $0.339_{-0.036}^{+0.037}$ & $0.318_{-0.036}^{+0.038}$ \\
\hline$\sigma_{8}$ & $0.89_{-0.16}^{+0.15}$ & $0.760_{-0.070}^{+0.074}$ & $0.811_{-0.077}^{+0.072}$ & $0.762_{-0.063}^{+0.066}$ & $0.755_{-0.064}^{+0.068}$ & $0.774_{-0.066}^{+0.066}$ \\
\hline$H_{0}[\mathrm{~km} / \mathrm{s} / \mathrm{Mpc}]$ & $>68$ & $68.7_{-2.5}^{+2.7}$ & $74.2_{-2.7}^{+2.8}$ & $68.3_{-1.9}^{+2.0}$ & $68.3_{-1.5}^{+1.6}$ & $70.3_{-1.7}^{+1.7}$ \\
\hline$S_{8}$ & $0.756_{-0.063}^{+0.064}$ & $0.802_{-0.036}^{+0.036}$ & $0.790_{-0.041}^{+0.040}$ & $0.808_{-0.039}^{+0.039}$ & $0.801_{-0.035}^{+0.037}$ & $0.796_{-0.040}^{+0.040}$ \\
\hline
\end{tabular}
$\mathrm{CMB}+$ Pantheon $+\mathrm{CC}, \mathrm{CMB}+\mathrm{BAO}+$ Pantheon $+\mathrm{CC}$, and CMB + R19 + Pantheon + CC, respectively. 


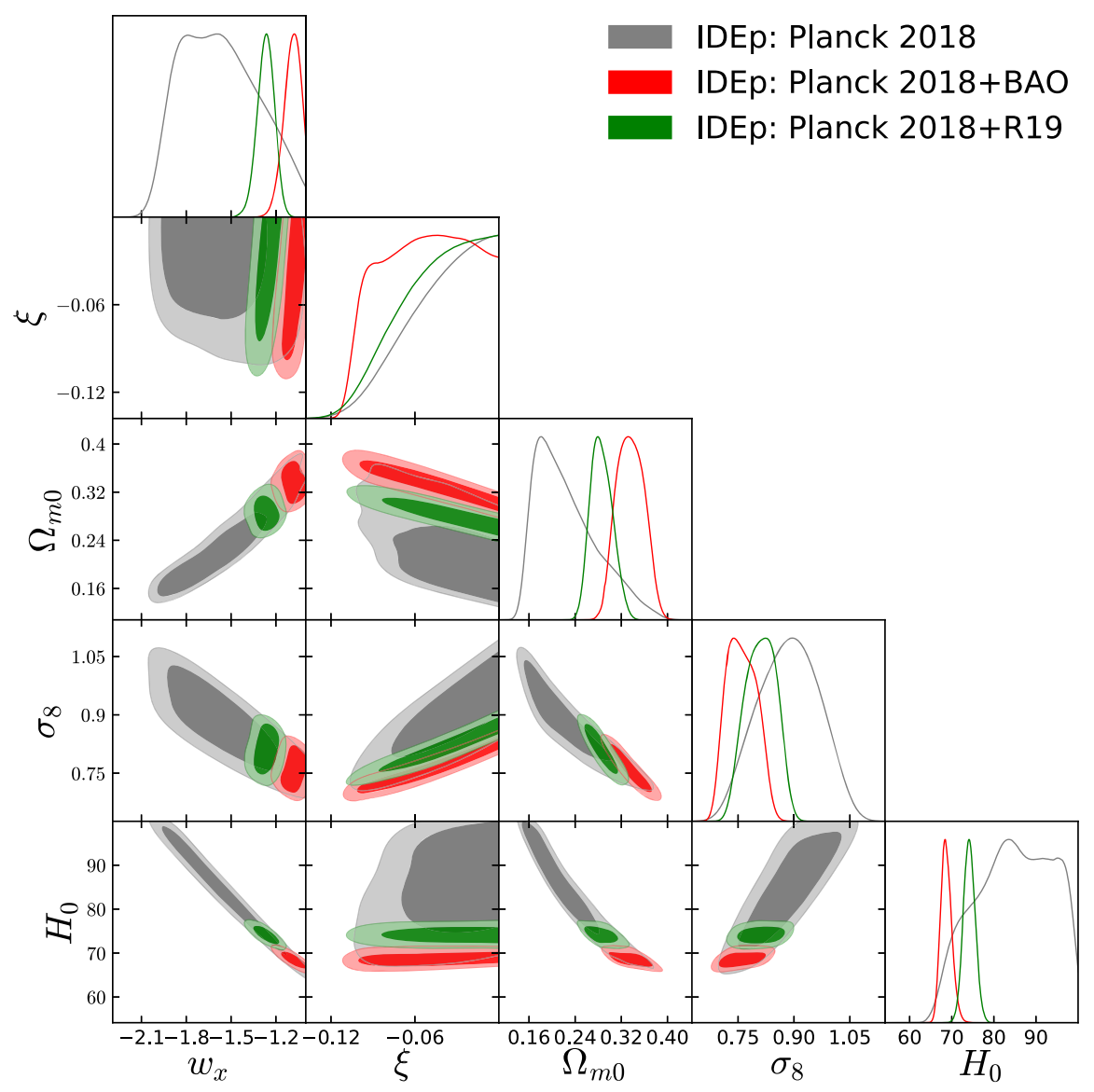

FIG. 1. $68 \%$ and 95\% C.L. allowed contours and one-dimensional posterior probability distributions within the minimal IDE model in the phantom-like dark energy scenario $\left(w_{x}<-1\right)$ from several data set combinations. Only a subset of cosmological parameters are shown.

dark energy $\left(w_{x}=-1.27_{-0.17}^{+0.14}\right.$ at $99 \%$ C.L. $)$. Adding the Pantheon and CC measurements to the CMB (see the fifth column of Table II) leads to constraints that are very similar to the $\mathrm{CMB}+\mathrm{BAO}$ case. The combination $\mathrm{CMB}+\mathrm{BAO}+$ Pantheon + CC (see the sixth column of Table II) only diminishes the error bars when compared to the previous case. Finally, the data combination of $\mathrm{CMB}+\mathrm{R} 19+$ Pantheon + CC (see the last column of Table II) is completely driven by the discrepancies between the $\mathrm{CMB}+$ Pantheon + CC data combination and the R19 measurements. Therefore, the Hubble constant shifts slightly towards higher values, reducing the tension with R19 at $2.3 \sigma$ and leading to evidence for a phantom dark energy component $w_{x}<-1$ at a high significance.

\section{IDE $+M_{\nu}:$ Nine parameters}

The results for an IDE $+M_{\nu}$ scenario, based on nine parameters, with a dark energy equation of state $w_{x}<-1$ are shown in Table III and Fig. 2.

In this scenario-extended to include the total neutrino mass $M_{\nu}$-independently of the data combination chosen, all of the constraints on the six cosmological parameters of the $\Lambda \mathrm{CDM}$ model as well as on the dark energy equation of state $w_{x}$ and all the derived parameters $\left(H_{0}, \Omega_{m 0}, \sigma_{8}\right.$, and
$S_{8}$ ) are very similar to those in the minimal IDE scenario of the previous section (see Table II). Therefore, the addition of the total neutrino mass does not introduce new correlations between the previous cosmological parameters, as we can see in Fig. 2. The well-known correlations between $M_{\nu}$ and the dark energy equation of state $w_{x}$, the matter density $\Omega_{m 0}$, and the clustering parameter $\sigma_{8}$ are present in this model, and are especially visible for the $\mathrm{CMB}+\mathrm{BAO}$ and $\mathrm{CMB}+\mathrm{R} 19$ data combinations. However, the also very well-known degeneracy between $H_{0}$ and $M_{\nu}$ disappears completely in this scenario. For this reason, the $M_{\nu}$ and $\Omega_{\nu} h^{2}$ upper limits are identical in the CMB and $\mathrm{CMB}+\mathrm{R} 19$ cases, instead of being improved when R19 measurements are added to $\mathrm{CMB}$ observations (as it happens in the $\Lambda \mathrm{CDM}+M_{\nu}$ model). Comparing the constraints obtained in this extended IDE scenario with those obtained within a $\Lambda \mathrm{CDM}$ scenario for the same combination of data sets, one can notice that the upper limits on $M_{\nu}$ are slightly relaxed in this case, because of the phantom behavior of the dark energy equation of state and the $M_{\nu}-w_{x}$ correlation. Cosmological neutrino mass bounds are softened if the dark energy equation of state is taken as a free parameter. If $w_{x}$ is allowed to vary, the matter energy density takes very high values, and this can 
TABLE III. $\quad 95 \%$ C.L. constraints within the IDE $+M_{\nu}$ model in the phantom-like dark energy scenario $\left(w_{x}<-1\right)$ from several data set combinations, where CB, CR19, CPCC, CBPCC, and CR19PCC correspond to the combinations CMB + BAO, CMB + R19, $\mathrm{CMB}+$ Pantheon $+\mathrm{CC}, \mathrm{CMB}+\mathrm{BAO}+$ Pantheon $+\mathrm{CC}$, and CMB + R19 + Pantheon + CC, respectively.

\begin{tabular}{|c|c|c|c|c|c|c|}
\hline Parameters & $\mathrm{CMB}$ & $\mathrm{CB}$ & CR19 & СРCC & CBPCC & CR19PCC \\
\hline$\Omega_{c} h^{2}$ & $0.133_{-0.015}^{+0.018}$ & $0.136_{-0.016}^{+0.015}$ & $0.135_{-0.015}^{+0.017}$ & $0.135_{-0.014}^{+0.014}$ & $0.135_{-0.015}^{+0.015}$ & $0.134_{-0.015}^{+0.016}$ \\
\hline$\Omega_{b} h^{2}$ & $0.02237_{-0.00031}^{+0.000030}$ & $0.02238_{-0.00028}^{+0.00028}$ & $0.02236_{-0.00031}^{+0.00031}$ & $0.02235_{-0.00029}^{+0.00030}$ & $0.02240_{-0.00027}^{+0.00028}$ & $0.02243_{-0.00029}^{+0.00029}$ \\
\hline $100 \theta_{M C}$ & $1.0402_{-0.0011}^{+0.0010}$ & $1.0401_{-0.0010}^{+0.00010}$ & $1.0401_{-0.0010}^{+0.0010}$ & $1.0401_{-0.0010}^{+0.0010}$ & $1.0401_{-0.0010}^{+0.0010}$ & $1.0402_{-0.0011}^{+0.0011}$ \\
\hline$\tau$ & $0.054_{-0.015}^{+0.016}$ & $0.055_{-0.015}^{+0.016}$ & $0.054_{-0.015}^{+0.016}$ & $0.054_{-0.015}^{+0.016}$ & $0.055_{-0.015}^{+0.016}$ & $0.055_{-0.015}^{+0.017}$ \\
\hline$n_{s}$ & $0.9650_{-0.0088}^{+0.0088}$ & $0.9654_{-0.0078}^{+0.0080}$ & $0.9646_{-0.0091}^{+0.0087}$ & $0.9645_{-0.0086}^{+0.0086}$ & $0.9660_{-0.0079}^{+0.0080}$ & $0.9663_{-0.0085}^{+0.0085}$ \\
\hline $\ln \left(10^{10} A_{s}\right)$ & $3.043_{-0.031}^{+0.033}$ & $3.045_{-0.031}^{+0.034}$ & $3.044_{-0.032}^{+0.033}$ & $3.045_{-0.031}^{+0.032}$ & $3.045_{-0.031}^{+0.033}$ & $3.045_{-0.031}^{+0.035}$ \\
\hline$w_{x}$ & $-1.60_{-0.46}^{+0.52}$ & $>-1.22$ & $-1.29_{-0.16}^{+0.15}$ & $-1.092_{-0.086}^{+0.090}$ & $>-1.15$ & $-1.136_{-0.087}^{+0.082}$ \\
\hline$\xi$ & $>-0.086$ & $>-0.101$ & $>-0.098$ & $>-0.102$ & $>-0.101$ & $>-0.100$ \\
\hline$\Omega_{m 0}$ & $0.23_{-0.09}^{+0.11}$ & $0.337_{0.043}^{+0.045}$ & $0.288_{-0.037}^{+0.039}$ & $0.342_{-0.042}^{+0.042}$ & $0.339_{-0.037}^{+0.037}$ & $0.318_{-0.038}^{+0.039}$ \\
\hline$\sigma_{8}$ & $0.88_{-0.14}^{+0.15}$ & $0.758_{-0.070}^{+0.076}$ & $0.803_{-0.076}^{+0.079}$ & $0.756_{-0.065}^{+0.071}$ & $0.756_{-0.065}^{+0.070}$ & $0.775_{-0.069}^{+0.070}$ \\
\hline$H_{0}[\mathrm{~km} / \mathrm{s} / \mathrm{Mpc}]$ & $>68$ & $68.8_{-2.7}^{+2.8}$ & $74.2_{-2.7}^{+2.7}$ & $68.2_{-2.1}^{+2.1}$ & $68.3_{-1.5}^{+1.5}$ & $70.4_{-1.7}^{+1.7}$ \\
\hline$M_{\nu}[\mathrm{eV}]$ & $<0.313$ & $<0.183$ & $<0.313$ & $<0.255$ & $<0.156$ & $<0.151$ \\
\hline$\Omega_{\nu} h^{2}$ & $<0.0034$ & $<0.0020$ & $<0.0034$ & $<0.0027$ & $<0.0017$ & $<0.0016$ \\
\hline$S_{8}$ & $0.754_{-0.064}^{+0.069}$ & $0.802_{-0.037}^{+0.039}$ & $0.785_{-0.045}^{+0.044}$ & $0.806_{-0.041}^{+0.042}$ & $0.802_{-0.037}^{+0.038}$ & $0.796_{-0.040}^{+0.042}$ \\
\hline
\end{tabular}

be compensated with a larger neutrino mass. Indeed, the authors of Ref. [67] found that a larger neutrino mass would be allowed when a dark coupling is present. The most stringent limit we find on the total neutrino mass is for the
$\mathrm{CMB}+\mathrm{R} 19+$ Pantheon $+\mathrm{CC}$ data combination, $M_{\nu}<$ $0.151 \mathrm{eV}$ at $95 \%$ C.L. The former bound is very similar to the one obtained for $\mathrm{CMB}+\mathrm{BAO}+$ Pantheon $+\mathrm{CC}$, $M_{\nu}<0.156 \mathrm{eV}$ at $95 \%$ C.L.

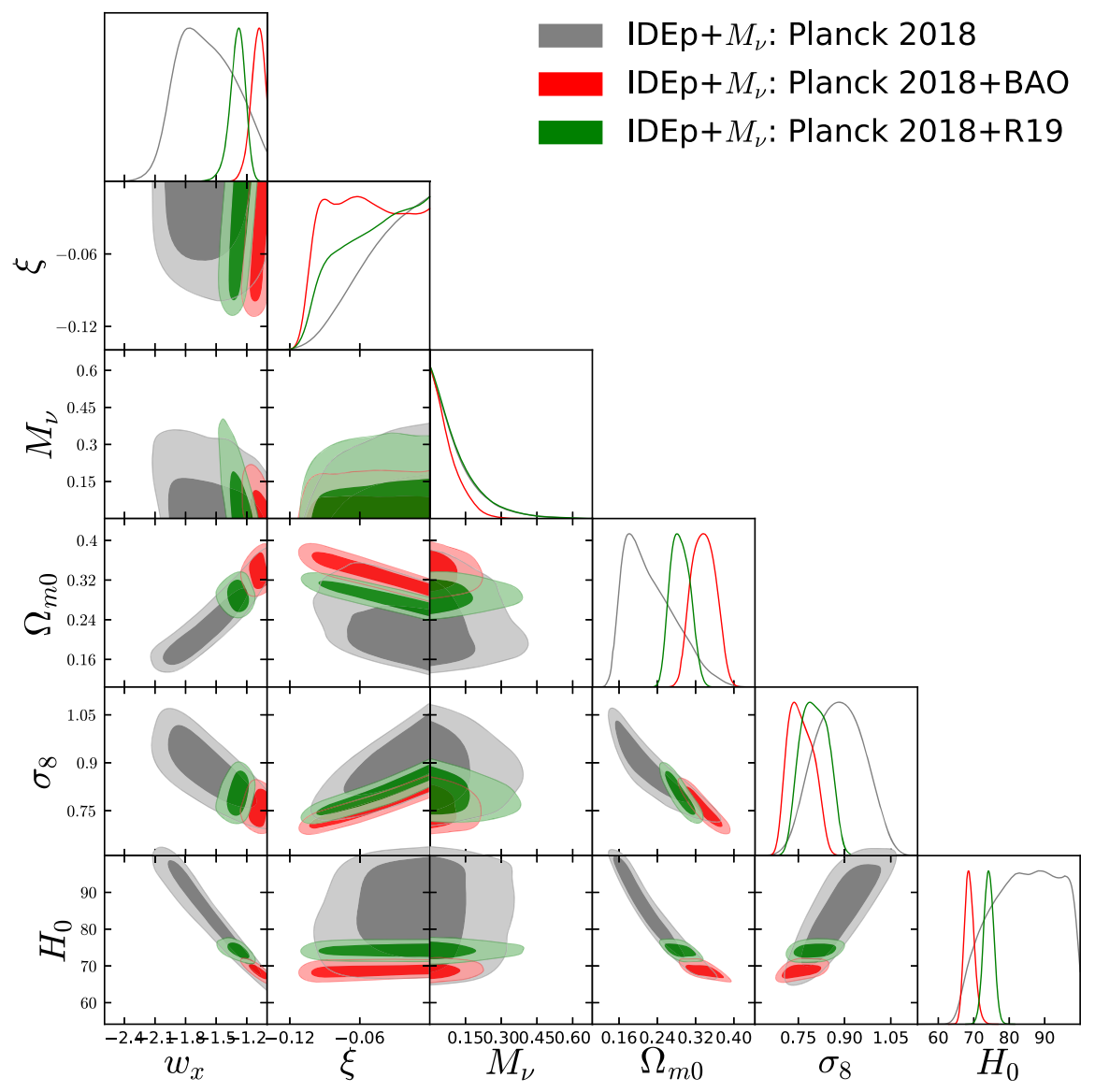

FIG. 2. $68 \%$ and $95 \%$ C.L. allowed contours and one-dimensional posterior probability distributions within the IDE $+M_{\nu}$ model in the phantom-like dark energy scenario $\left(w_{x}<-1\right)$ from several data set combinations. Only a subset of cosmological parameters are shown. 
TABLE IV. $95 \%$ C.L. constraints within the IDE $+N_{\text {eff }}$ scheme in the phantom-like dark energy scenario $\left(w_{x}<-1\right)$ from several data set combinations, where CB, CR19, CPCC, CBPCC, and CR19PCC correspond to the combinations CMB $+\mathrm{BAO}, \mathrm{CMB}+\mathrm{R} 19$, $\mathrm{CMB}+$ Pantheon $+\mathrm{CC}, \mathrm{CMB}+\mathrm{BAO}+$ Pantheon $+\mathrm{CC}$, and CMB + R19 + Pantheon + CC, respectively.

\begin{tabular}{|c|c|c|c|c|c|c|}
\hline Parameters & $\mathrm{CMB}$ & $\mathrm{CB}$ & CR19 & CPCC & CBPCC & CR19PCC \\
\hline$\Omega_{c} h^{2}$ & $0.131_{-0.017}^{+0.019}$ & $0.133_{-0.017}^{+0.017}$ & $0.131_{-0.016}^{+0.018}$ & $0.134_{-0.017}^{+0.017}$ & $0.135_{-0.017}^{+0.017}$ & $0.138_{-0.017}^{+0.018}$ \\
\hline$\Omega_{b} h^{2}$ & $0.02227_{-0.00043}^{+0.00042}$ & $0.02230_{-0.00039}^{+0.00039}$ & $0.02226_{-0.00043}^{+0.00043}$ & $0.02232_{-0.00039}^{+0.00038}$ & $0.02238_{-0.00035}^{+0.00036}$ & $0.02255_{-0.00036}^{+0.00037}$ \\
\hline $100 \theta_{M C}$ & $1.0404_{-0.0013}^{+0.0012}$ & $1.0404_{-0.0013}^{+0.0012}$ & $1.0404_{-0.0013}^{+0.0012}$ & $1.0402_{-0.0012}^{+0.0012}$ & $1.0402_{-0.0012}^{+0.0012}$ & $1.0399_{-0.0012}^{+0.0012}$ \\
\hline$\tau$ & $0.053_{-0.016}^{+0.016}$ & $0.054_{-0.015}^{+0.016}$ & $0.053_{-0.015}^{+0.016}$ & $0.054_{-0.015}^{+0.016}$ & $0.055_{-0.015}^{+0.016}$ & $0.056_{-0.015}^{+0.017}$ \\
\hline$n_{s}$ & $0.960_{-0.017}^{+0.017}$ & $0.962_{-0.015}^{+0.015}$ & $0.960_{-0.017}^{+0.017}$ & $0.963_{-0.015}^{+0.015}$ & $0.965_{-0.014}^{+0.014}$ & $0.972_{-0.013}^{+0.013}$ \\
\hline $\ln \left(10^{10} A_{s}\right)$ & $3.037_{-0.038}^{+0.017}$ & $3.039_{-0.036}^{+0.036}$ & $3.036_{-0.037}^{+0.037}$ & $3.042_{-0.036}^{+0.036}$ & $3.043_{-0.035}^{+0.036}$ & $3.053_{-0.034}^{+0.036}$ \\
\hline$w_{x}$ & $-1.61_{-0.46}^{+0.54}$ & $>-1.22$ & $-1.31_{-0.15}^{+0.14}$ & $-1.090_{-0.079}^{+0.087}$ & $-1.082_{-0.074}^{+0.081}$ & $-1.122_{-0.088}^{+0.084}$ \\
\hline$\xi$ & $>-0.087$ & $>-0.099$ & $>-0.094$ & $>-0.101$ & $>-0.101$ & $>-0.101$ \\
\hline$\Omega_{m 0}$ & $0.22_{-0.09}^{+0.12}$ & $0.334_{-0.042}^{+0.046}$ & $0.281_{-0.037}^{+0.039}$ & $0.340_{-0.041}^{+0.040}$ & $0.339_{-0.036}^{+0.036}$ & $0.321_{-0.036}^{+0.037}$ \\
\hline$\sigma_{8}$ & $0.89_{-0.16}^{+0.15}$ & $0.761_{-0.071}^{+0.074}$ & $0.814_{-0.075}^{+0.072}$ & $0.758_{-0.064}^{+0.068}$ & $0.754_{-0.064}^{+0.068}$ & $0.773_{-0.069}^{+0.070}$ \\
\hline$H_{0}[\mathrm{~km} / \mathrm{s} / \mathrm{Mpc}]$ & $>67$ & $68.4_{-2.9}^{+3.2}$ & $74.2_{-2.8}^{+2.7}$ & $68.0_{-2.5}^{+2.6}$ & $68.2_{-2.1}^{+2.1}$ & $70.9_{-2.0}^{+2.0}$ \\
\hline$N_{\text {eff }}$ & $2.91_{-0.38}^{+0.38}$ & $2.94_{-0.35}^{+0.36}$ & $2.90_{-0.36}^{+0.38}$ & $2.99_{-0.31}^{+0.32}$ & $3.02_{-0.31}^{+0.1}$ & $3.22_{-0.29}^{+0.31}$ \\
\hline$S_{8}$ & $0.753_{-0.065}^{+0.068}$ & $0.801_{-0.035}^{+0.036}$ & $0.785_{-0.041}^{+0.0041}$ & $0.806_{-0.040}^{+0.040}$ & $0.800_{-0.035}^{+0.037}$ & $0.798_{-0.040}^{+0.041}$ \\
\hline
\end{tabular}

\section{IDE $+N_{\mathrm{eff}}:$ Nine parameters}

The results for an IDE $+N_{\text {eff }}$ scenario, based on nine parameters, with a dark energy equation of state $w_{x}<-1$ are shown in Table IV and Fig. 3. Notice that in general the constraints on the cosmological parameters of the $\Lambda \mathrm{CDM}$ model, with the exception of the spectral index $n_{s}$, are very similar to those obtained in previous scenarios. Due to the well-known correlation between $N_{\text {eff }}$ and $n_{s}$, we notice a

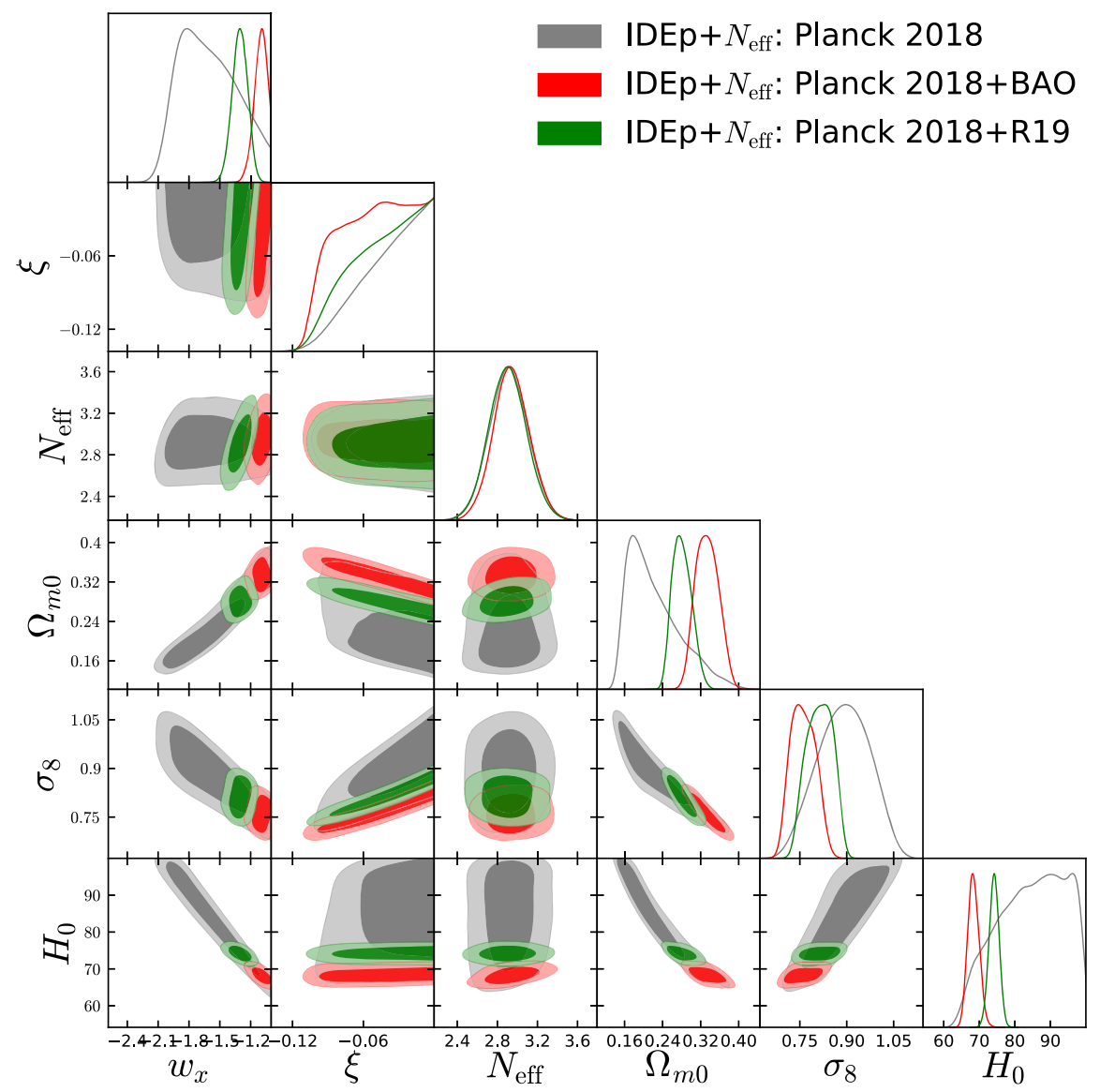

FIG. 3. $68 \%$ and $95 \%$ C.L. allowed contours and one-dimensional posterior probability distributions within the IDE $+N_{\text {eff }}$ model in the phantom-like dark energy scenario $\left(w_{x}<-1\right)$ from several data set combinations. Only a subset of cosmological parameters are shown. 
very mild shift in the mean value of the spectral index toward smaller values. The effective number of relativistic degrees of freedom $N_{\text {eff }}$ we recover in this modified cosmological scenario is perfectly consistent with its standard value $N_{\text {eff }}=3.046[68,69]$. Also in this scenario, the addition of the effective number of neutrino species $N_{\text {eff }}$ as a free parameter does not introduce a new direction of correlation between the previous cosmological parameters; see Fig. 3. The well-known correlation between $N_{\text {eff }}$ and the Hubble constant $H_{0}$ disappears completely in this scenario, since the dark energy equation of state is also an additional free parameter. For this very same reason, the constraints on $N_{\text {eff }}$ are the same for the $\mathrm{CMB}$ and $\mathrm{CMB}+\mathrm{R} 19$ cases. When adding Pantheon and CC data to the CMB (see the fifth column of Table IV), the relativistic degrees of freedom shift towards higher values $\left(N_{\text {eff }}=2.99 \pm 0.16\right.$ at $68 \%$ C.L. $)$ with respect to the CMB-only case (where $N_{\text {eff }}=2.91 \pm$ 0.019 at $68 \%$ C.L.). When we further add the R19 measurements, i.e., we consider CMB + R19 + Pantheon + $\mathrm{CC}$ (see the last column of Table IV), we obtain our most stringent bound $N_{\text {eff }}=3.22_{-0.29}^{+0.31}$ at $95 \%$ C.L. associated with the highest mean value for $N_{\text {eff }}$.

\section{IDE $+M_{\nu}+N_{\mathrm{eff}}:$ Ten parameters}

The results for an IDE $+M_{\nu}+N_{\text {eff }}$ scenario, based on ten parameters, with a dark energy equation of state $w_{x}<$ -1 are shown in Table V and Fig. 4. In this scenario the spectral index $n_{s}$ is shifted further down and we obtain an indication that $w_{x}$ is a phantom at a high significance for the $\mathrm{CMB}+\mathrm{R} 19$ data set combination; see Table V.

The considerations we made in the previous cases about the directions of the correlations between the cosmological parameters are unaltered by the variation of $M_{\nu}$ and $N_{\text {eff }}$ at the same time. While the constraints on the effective number of neutrino species $N_{\text {eff }}$ for all data set combinations are identical to the IDE $+N_{\text {eff }}$ scenario, the upper limits on the total neutrino mass $M_{\nu}$ are mildly relaxed with respect to the IDE $+M_{\nu}$ model. The most stringent bound on this parameter is now obtained for the combination $\mathrm{CMB}+\mathrm{BAO}+$ Pantheon + CC (see the sixth column of Table V), and it corresponds to $M_{\nu}<0.160 \mathrm{eV}$ at $95 \%$ C.L.

\section{B. IDE with $w_{x}>-1$}

In the following sections we present the constraints for the case of a quintessence-like dark energy equation of state $w_{x}>-1$.

\section{IDE}

The results for our baseline IDE scenario, based on eight parameters, with a dark energy equation of state $w_{x}>-1$ are shown in Table VI and Fig. 5.

Notice that the amount of cold dark matter is much lower than that in the canonical $\Lambda \mathrm{CDM}$ scenario. The reason for this is due to the energy flow from the DM to DE sector and, consequently, the current amount of dark matter energy density decreases as $\xi$ increases; see Fig. 5 and Refs. $[58,70]$. For this very same reason the CMB + R19 data combination prefers a nonzero value of the coupling $\xi$ at a very high significance.

For the CMB-only case (see the second column of Table VI), the Hubble constant is shifted towards a higher value, mildly alleviating the Hubble constant tension with $\mathrm{R} 19$ data at $1.1 \sigma$ (mainly via the degeneracy between $\xi$ and $H_{0}$ ), as can be seen in Fig. 5. When including the BAO data (see the third column of Table VI), most of the parameters

TABLE V. $95 \%$ C.L. constraints within the IDE $+M_{\nu}+N_{\text {eff }}$ scheme in the phantom-like dark energy scenario $\left(w_{x}<-1\right)$ from several data set combinations, where CB, CR19, CPCC, CBPCC, and CR19PCC correspond to the combinations CMB + BAO, $\mathrm{CMB}+\mathrm{R} 19, \mathrm{CMB}+$ Pantheon $+\mathrm{CC}, \mathrm{CMB}+\mathrm{BAO}+$ Pantheon + CC, and CMB + R19 + Pantheon + CC, respectively.

\begin{tabular}{|c|c|c|c|c|c|c|}
\hline Parameters & CMB & $\mathrm{CB}$ & CR19 & $\mathrm{CPCC}$ & $\mathrm{CBPCC}$ & CR19PCC \\
\hline$\Omega_{c} h^{2}$ & $0.132_{-0.017}^{+0.019}$ & $0.134_{-0.018}^{+0.017}$ & $0.132_{-0.017}^{+0.018}$ & $0.135_{-0.017}^{+0.017}$ & $0.135_{-0.017}^{+0.017}$ & $0.139_{-0.018}^{+0.018}$ \\
\hline$\Omega_{b} h^{2}$ & $0.02223_{-0.00045}^{+0.00046}$ & $0.02230_{-0.00040}^{+0.00042}$ & $0.02223_{-0.00044}^{+0.00043}$ & $0.02232_{-0.00038}^{+0.00038}$ & $0.02237_{-0.00036}^{+0.00036}$ & $0.02256_{-0.00035}^{+0.00036}$ \\
\hline $100 \theta_{M C}$ & $1.0404_{-0.0013}^{+0.0013}$ & $1.0403_{-0.0012}^{+0.0013}$ & $1.0404_{-0.0012}^{+0.0012}$ & $1.0402_{-0.0012}^{+0.0012}$ & $1.0402_{-0.0012}^{+0.0012}$ & $1.0399_{-0.0012}^{+0.0012}$ \\
\hline$\tau$ & $0.053_{-0.016}^{+0.016}$ & $0.054_{-0.015}^{+0.016}$ & $0.053_{-0.015}^{+0.017}$ & $0.054_{-0.015}^{+0.016}$ & $0.055_{-0.015}^{+0.017}$ & $0.056_{-0.016}^{+0.017}$ \\
\hline$n_{s}$ & $0.959_{-0.017}^{+0.017}$ & $0.962_{-0.015}^{+0.016}$ & $0.959_{-0.016}^{+0.017}$ & $0.963_{-0.015}^{+0.015}$ & $0.965_{-0.014}^{+0.014}$ & $0.973_{-0.013}^{+0.013}$ \\
\hline $\ln \left(10^{10} A_{s}\right)$ & $3.036_{-0.037}^{+0.037}$ & $3.040_{-0.036}^{+0.037}$ & $3.037_{-0.037}^{+0.039}$ & $3.043_{-0.034}^{+0.036}$ & $3.043_{-0.034}^{+0.037}$ & $3.054_{-0.035}^{+0.037}$ \\
\hline$w_{x}$ & $>-2.13$ & $>-1.23$ & $-1.34_{0.20}^{+0.19}$ & $-1.097_{-0.088}^{+0.091}$ & $>-1.158$ & $-1.123_{-0.093}^{+0.089}$ \\
\hline$\xi$ & $>-0.093$ & $>-0.101$ & $>-0.097$ & $>-0.101$ & $>-0.101$ & $>-0.102$ \\
\hline$\Omega_{m 0}$ & $0.23_{-0.10}^{+0.13}$ & $0.336_{-0.042}^{+0.044}$ & $0.283_{-0.037}^{+0.040}$ & $0.342_{-0.040}^{+0.041}$ & $0.339_{-0.037}^{+0.037}$ & $0.322_{-0.037}^{+0.038}$ \\
\hline$\sigma_{8}$ & $0.87_{-0.16}^{+0.16}$ & $0.757_{-0.068}^{+0.074}$ & $0.805_{-0.075}^{+0.079}$ & $0.755_{-0.064}^{+0.069}$ & $0.756_{-0.065}^{+0.071}$ & $0.772_{-0.069}^{+0.075}$ \\
\hline$H_{0}[\mathrm{~km} / \mathrm{s} / \mathrm{Mpc}]$ & $>65$ & $68.4_{-3.1}^{+3.1}$ & $74.2_{-2.7}^{+2.8}$ & $68.0_{-2.7}^{+2.7}$ & $68.2_{-2.1}^{+2.2}$ & $70.9_{-2.0}^{+1.9}$ \\
\hline$M_{\nu}[\mathrm{eV}]$ & $<0.367$ & $<0.184$ & $<0.332$ & $<0.263$ & $<0.160$ & $<0.172$ \\
\hline$N_{\text {eff }}$ & $2.89_{-0.37}^{+0.37}$ & $2.94_{-0.35}^{+0.39}$ & $2.88_{-0.36}^{+0.37}$ & $3.00_{-0.33}^{+0.34}$ & $3.02_{-0.31}^{+0.33}$ & $3.22_{-0.29}^{+0.29}$ \\
\hline$\Omega_{\nu} h^{2}$ & $<0.0037$ & $<0.0019$ & $<0.0034$ & $<0.0028$ & $<0.0017$ & $<0.0018$ \\
\hline$S_{8}$ & $0.751_{-0.067}^{+0.068}$ & $0.800_{-0.037}^{+0.039}$ & $0.779_{-0.045}^{+0.044}$ & $0.805_{-0.040}^{+0.041}$ & $0.803_{-0.037}^{+0.039}$ & $0.798_{-0.041}^{+0.042}$ \\
\hline
\end{tabular}




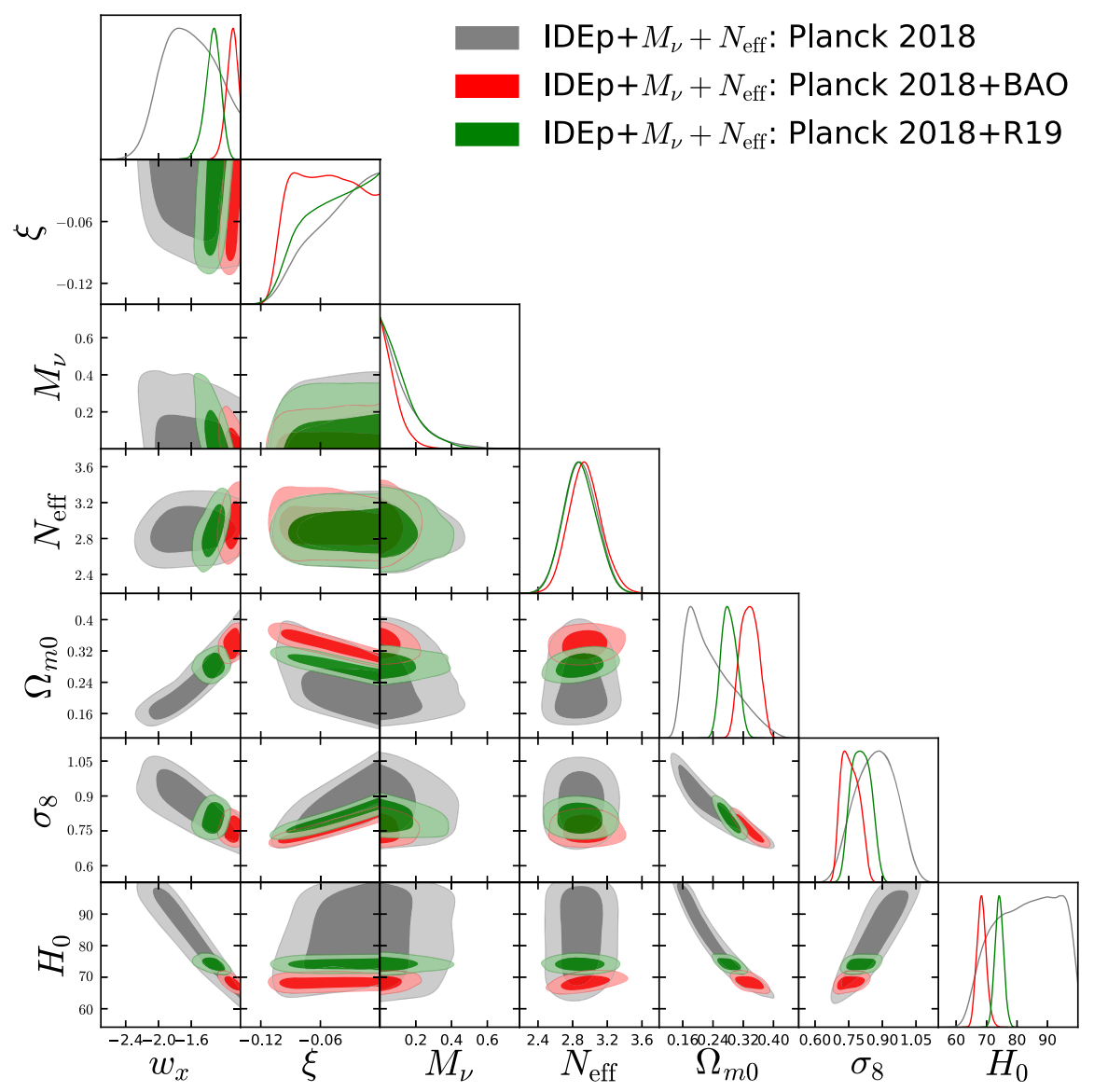

FIG. 4. $68 \%$ and $95 \%$ C.L. allowed contours and one-dimensional posterior probability distributions within the IDE $+M_{\nu}+N_{\text {eff }}$ model in the phantom-like dark energy scenario $\left(w_{x}<-1\right)$ from several data set combinations. Only a subset of cosmological parameters are shown.

are unmodified with respect to the CMB-only case. The tension on $H_{0}$ with R19 data is at the $2.9 \sigma$ level. While the values of $\sigma_{8}$ and $S_{8}$ go down with respect to the CMB-only case, they are still far from those corresponding to the $\Lambda \mathrm{CDM}$ model, even if they are in agreement due to the very large error bars.

TABLE VI. $\quad 95 \%$ C.L. constraints within the IDE model in the quintessence-like dark energy scenario $\left(w_{x}>-1\right)$ from several data set combinations, where $\mathrm{CB}, \mathrm{CR} 19, \mathrm{CPCC}, \mathrm{CBPCC}$, and CR19PCC correspond to the combinations $\mathrm{CMB}+\mathrm{BAO}$, CMB $+\mathrm{R} 19$, $\mathrm{CMB}+$ Pantheon + CC, CMB + BAO + Pantheon + CC, and CMB + R19 + Pantheon + CC, respectively.

\begin{tabular}{|c|c|c|c|c|c|c|}
\hline Parameters & CMB & $\mathrm{CB}$ & CR19 & $\mathrm{CPCC}$ & CBPCC & CR19PCC \\
\hline$\overline{\Omega_{c} h^{2}}$ & $<0.115$ & $0.076_{-0.058}^{+0.046}$ & $<0.055$ & $0.075_{-0.059}^{+0.046}$ & $0.077_{-0.059}^{+0.044}$ & $0.057_{-0.051}^{+0.040}$ \\
\hline$\Omega_{b} h^{2}$ & $0.02236_{-0.00029}^{+0.00029}$ & $0.02239_{-0.00027}^{+0.00029}$ & $0.02238_{-0.00030}^{+0.00030}$ & $0.02236_{-0.00028}^{+0.00029}$ & $0.02239_{-0.00027}^{+0.00028}$ & $0.02241_{-0.00029}^{+0.00029}$ \\
\hline $100 \theta_{M C}$ & $1.0448_{-0.0043}^{+0.0050}$ & $1.0438_{-0.0031}^{+0.0043}$ & $1.0476_{-0.0029}^{+0.0027}$ & $1.0438_{-0.0032}^{+0.0044}$ & $1.0437_{-0.0031}^{+0.0044}$ & $1.0450_{-0.0032}^{+0.0042}$ \\
\hline$\tau$ & $0.054_{-0.015}^{+0.016}$ & $0.055_{-0.015}^{+0.017}$ & $0.054_{-0.015}^{+0.015}$ & $0.054_{-0.015}^{+0.016}$ & $0.055_{-0.015}^{+0.016}$ & $0.055_{-0.015}^{+0.016}$ \\
\hline$n_{s}$ & $0.9650_{-0.0084}^{+0.0084}$ & $0.9658_{-0.0084}^{+0.0082}$ & $0.9659_{-0.0082}^{+0.0080}$ & $0.9648_{-0.0085}^{+0.0082}$ & $0.9660_{-0.0079}^{+0.0079}$ & $0.9661_{-0.0084}^{+0.0085}$ \\
\hline $\ln \left(10^{10} A_{s}\right)$ & $3.045_{-0.031}^{+0.031}$ & $3.045_{-0.032}^{+0.034}$ & $3.044_{-0.031}^{+0.031}$ & $3.045_{-0.031}^{+0.032}$ & $3.045_{-0.031}^{+0.033}$ & $3.045_{-0.031}^{+0.033}$ \\
\hline$w_{x}$ & $<-0.77$ & $<-0.80$ & $<-0.904$ & $<-0.79$ & $<-0.79$ & $<-0.834$ \\
\hline$\xi$ & $<0.27$ & $<0.24$ & $0.231_{-0.071}^{+0.060}$ & $<0.25$ & $<0.24$ & $0.16_{-0.10}^{+0.11}$ \\
\hline$\Omega_{m 0}$ & $0.18_{-0.15}^{+0.16}$ & $0.21_{-0.13}^{+0.11}$ & $0.089_{-0.054}^{+0.067}$ & $0.21_{-0.13}^{+0.11}$ & $0.22_{-0.13}^{+0.10}$ & $0.16_{-0.10}^{+0.09}$ \\
\hline$\sigma_{8}$ & $1.6_{-1.2}^{+2.1}$ & $1.3_{-0.7}^{+1.1}$ & $2.6_{-1.4}^{+1.8}$ & $1.3_{-0.7}^{+1.2}$ & $1.2_{-0.6}^{+1.1}$ & $1.6_{-0.9}^{+1.5}$ \\
\hline$H_{0}[\mathrm{~km} / \mathrm{s} / \mathrm{Mpc}]$ & $69.3_{-6.5}^{+6.2}$ & $68.4_{-2.5}^{+2.7}$ & $73.3_{-2.3}^{+2.3}$ & $68.2_{-1.9}^{+1.9}$ & $68.3_{-1.5}^{+1.6}$ & $70.2_{-1.6}^{+1.7}$ \\
\hline$S_{8}$ & $1.07_{-0.32}^{+0.52}$ & $0.97_{-0.21}^{+0.35}$ & $1.32_{-0.34}^{+0.39}$ & $0.98_{-0.22}^{+0.96}$ & $0.97_{-0.20}^{+0.35}$ & $1.06_{-0.26}^{+1.0}$ \\
\hline
\end{tabular}




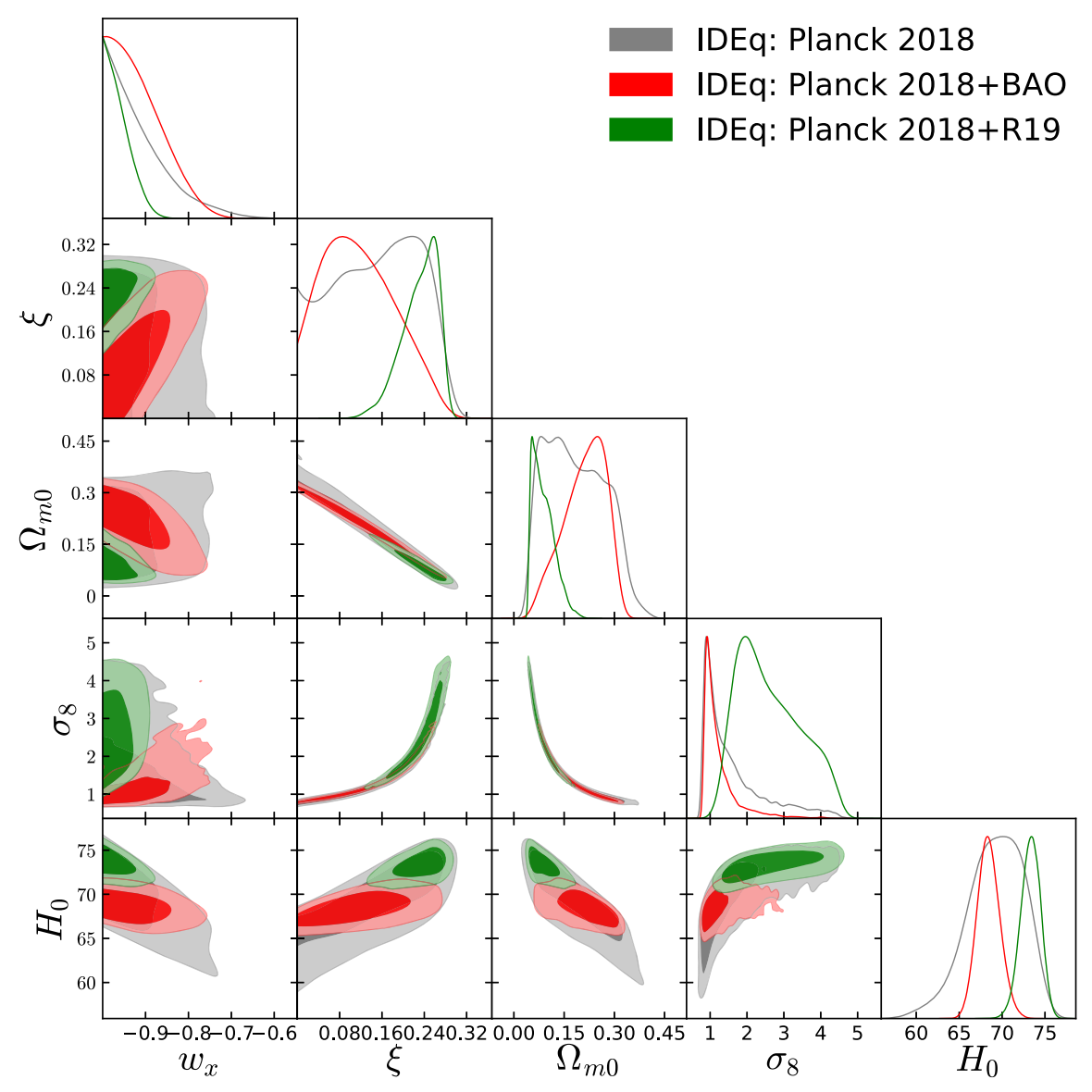

FIG. 5. $68 \%$ and 95\% C.L. allowed contours and one-dimensional posterior probability distributions within the IDE model in the quintessence-like dark energy scenario $\left(w_{x}>-1\right)$ from several data set combinations. Only a subset of cosmological parameters are shown.

When considering the $\mathrm{CMB}+\mathrm{R} 19$ combination (see the fourth column of Table VI), the Hubble constant value moves in agreement with the R19 measurement within $1 \sigma$, leading to a stronger upper limit on the dark energy equation of state $w_{x}$ and pushing the value towards the cosmological constant case. In particular, we have $w<$ -0.904 at $95 \%$ C.L. for CMB + R19. As previously stated, we find a mean value of the coupling $\xi$ that is different from zero at many standard deviations, due to the flux of energy from the DM to DE sector. Both $\sigma_{8}$ and $S_{8}$ move towards extremely high values with very large error bars, increasing the tension between Planck and the cosmic shear data. The addition of Pantheon and CC to CMB measurements (fifth column of Table VI) leads to a Hubble constant disagreement with R19 data at the $3 \sigma$ level. The data set combination $\mathrm{CMB}+\mathrm{R} 19+$ Pantheon $+\mathrm{CC}$ provides constraints that lie between the $\mathrm{CMB}+$ Pantheon $+\mathrm{CC}$ and $\mathrm{CMB}+\mathrm{R} 19$ cases, slightly shifting the Hubble constant towards higher values (and therefore reducing the tension with R19 data down to the $2.4 \sigma$ level) and setting limits on the coupling $\xi$ that are different from zero at a high significance.

\section{IDE $+M_{\nu}:$ Nine parameters}

The results for an IDE $+M_{\nu}$ scenario, based on nine parameters, with a dark energy equation of state $w_{x}>-1$ are shown in Table VII and Fig. 6. The addition of a varying neutrino mass does not change the directions of the correlations between the cosmological parameters we have without $M_{\nu}$, as can be seen in Fig. 6. The well-known correlation between $M_{\nu}$ and the dark energy equation of state $w_{x}$ is present also in this model, while the negative degeneracy present between $H_{0}$ and $M_{\nu}$ is completely absent in this scenario with $w_{x}>-1$. With respect to the phantom regime, a very mild correlation between $M_{\nu}$ and $\xi$ appears, especially in the case of the CMB + R19 data set combination, as we can see in Fig. 6. For this reason, the $M_{\nu}$ and $\Omega_{\nu} h^{2}$ upper limits improve in the CMB $+\mathrm{R} 19$ case, contrary to what happens when $w_{x}<-1$ (see Table III). Similarly to the phantom model case, the $M_{\nu}$ upper limits are relaxed, and the most stringent limit we find on the total neutrino mass is for the $\mathrm{CMB}+$ Pantheon $+\mathrm{CC}$ and $\mathrm{CMB}+\mathrm{R} 19+$ Pantheon+CC data combinations, for which $M_{\nu}<0.152 \mathrm{eV}$ at $95 \%$ C.L. 
TABLE VII. $\quad 95 \%$ C.L. constraints within the IDE $+M_{\nu}$ model in the quintessence-like dark energy scenario $\left(w_{x}>-1\right)$ from several data set combinations, where CB, CR19, CPCC, CBPCC, and CR19PCC correspond to the combinations CMB + BAO, CMB + R19, $\mathrm{CMB}+$ Pantheon $+\mathrm{CC}, \mathrm{CMB}+\mathrm{BAO}+$ Pantheon $+\mathrm{CC}$, and CMB + R19 + Pantheon + CC, respectively.

\begin{tabular}{|c|c|c|c|c|c|c|}
\hline Parameters & $\mathrm{CMB}$ & $\mathrm{CB}$ & CR19 & $\mathrm{CPCC}$ & CBPCC & CR19PCC \\
\hline$\Omega_{c} h^{2}$ & $<0.116$ & $0.073_{-0.058}^{+0.048}$ & $<0.056$ & $0.074_{-0.057}^{+0.046}$ & $0.076_{-0.058}^{+0.045}$ & $0.059_{-0.051}^{+0.041}$ \\
\hline$\Omega_{b} h^{2}$ & $0.02233_{-0.00032}^{+0.00031}$ & $0.02238_{-0.00028}^{+0.00028}$ & $0.02238_{-0.00029}^{+0.00030}$ & $0.02234_{-0.00031}^{+0.00030}$ & $0.02239_{-0.00028}^{+0.00028}$ & $0.02242_{-0.00027}^{+0.00029}$ \\
\hline $100 \theta_{M C}$ & $1.0448_{-0.0043}^{+0.0049}$ & $1.0439_{-0.0033}^{+0.0044}$ & $1.0476_{-0.0031}^{+0.0027}$ & $1.0438_{-0.0032}^{+0.00031}$ & $1.0437_{-0.0031}^{+0.0043}$ & $1.0449_{-0.0032}^{+0.0041}$ \\
\hline$\tau$ & $0.054_{-0.015}^{+0.016}$ & $0.055_{-0.015}^{+0.017}$ & $0.054_{-0.015}^{+0.016}$ & $0.054_{-0.016}^{+0.016}$ & $0.055_{-0.015}^{+0.016}$ & $0.055_{-0.015}^{+0.016}$ \\
\hline$n_{s}$ & $0.9644_{-0.0090}^{+0.0089}$ & $0.9660_{-0.0080}^{+0.0079}$ & $0.9657_{-0.0085}^{+0.0084}$ & $0.9646_{-0.0087}^{+0.0085}$ & $0.9660_{-0.0080}^{+0.0081}$ & $0.9665_{-0.0083}^{+0.0084}$ \\
\hline $\ln \left(10^{10} A_{s}\right)$ & $3.045_{-0.031}^{+0.032}$ & $3.045_{-0.031}^{+0.033}$ & $3.044_{-0.032}^{+0.033}$ & $3.045_{-0.031}^{+0.032}$ & $3.045_{-0.031}^{+0.033}$ & $3.045_{-0.031}^{+0.033}$ \\
\hline$w_{x}$ & $<-0.76$ & $<-0.79$ & $<-0.905$ & $<-0.80$ & $<-0.79$ & $<-0.835$ \\
\hline$\xi$ & $<0.28$ & $<0.25$ & $0.234_{-0.081}^{+0.067}$ & $<0.26$ & $<0.24$ & $0.158_{-0.10}^{+0.11}$ \\
\hline$\Omega_{m 0}$ & $0.19_{-0.13-0.15}^{+0.17}$ & $0.21_{-0.13}^{+0.11}$ & $0.089_{-0.054}^{+0.069}$ & $0.21_{-0.13}^{+0.11}$ & $0.21_{-0.13}^{+0.10}$ & $0.17_{-0.10}^{+0.09}$ \\
\hline$\sigma_{8}$ & $1.6_{-1.1}^{+2.0}$ & $1.3_{-0.7}^{+1.2}$ & $2.6_{-1.5}^{+1.8}$ & $1.3_{-0.7}^{+1.1}$ & $1.2_{-0.6}^{+1.1}$ & $1.5_{-0.8}^{+1.4}$ \\
\hline$H_{0}[\mathrm{~km} / \mathrm{s} / \mathrm{Mpc}]$ & $68.8_{-7.1}^{+6.6}$ & $68.5_{-2.5}^{+2.8}$ & $73.3_{-2.4}^{+2.2}$ & $68.1_{-2.1}^{+2.1}$ & $68.3_{-1.5}^{+1.6}$ & $70.2_{-1.6}^{+1.7}$ \\
\hline$M_{\nu}$ & $<0.318$ & $<0.173$ & $<0.203$ & $<0.263$ & $<0.152$ & $<0.152$ \\
\hline$\Omega_{\nu} h^{2}$ & $<0.0034$ & $<0.0019$ & $<0.0022$ & $<0.0028$ & $<0.0016$ & $<0.0016$ \\
\hline$S_{8}$ & $1.08_{-0.31}^{+0.51}$ & $0.99_{-0.22}^{+0.36}$ & $1.33_{-0.35}^{+0.39}$ & $0.98_{-0.21}^{+0.33}$ & $0.97_{-0.20}^{+0.34}$ & $1.05_{-0.25}^{+0.40}$ \\
\hline
\end{tabular}

\section{IDE $+N_{\mathrm{eff}}:$ Nine parameters}

Table VIII and Fig. 7 depict the results for an IDE $+N_{\text {eff }}$ scenario, based on nine parameters. Concerning the correlation between $N_{\text {eff }}$ and $n_{s}$, we notice a shift of $1 \sigma$ of the spectral index towards lower values, followed by a shift of $H_{0}$ in the same direction.

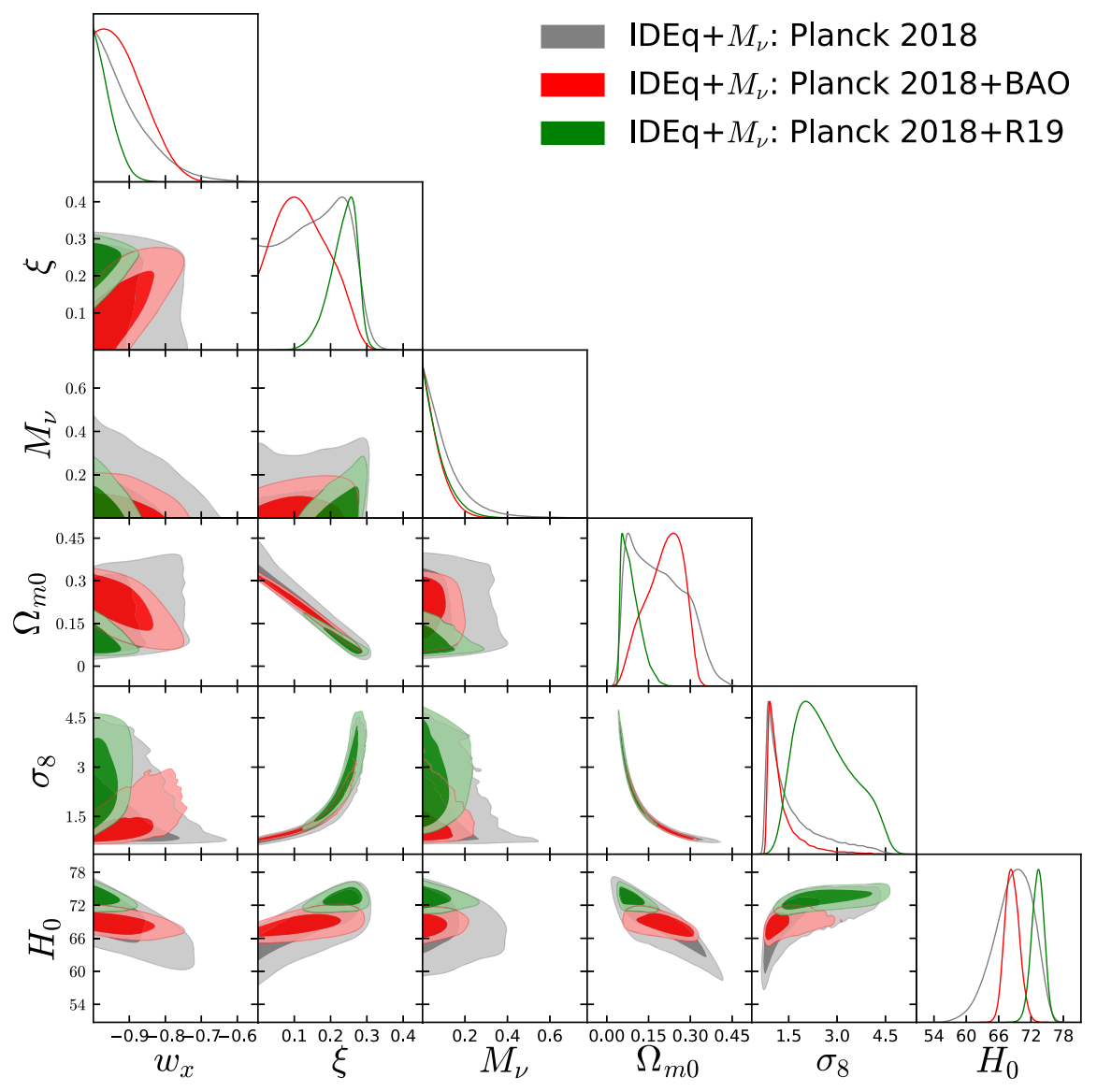

FIG. 6. $68 \%$ and $95 \%$ C.L. allowed contours and one-dimensional posterior probability distributions within the IDE $+M_{\nu}$ model in the quintessence-like dark energy scenario $\left(w_{x}>-1\right)$ from several data set combinations. Only a subset of cosmological parameters are shown. 
TABLE VIII. $\quad 95 \%$ C.L. constraints within the IDE $+N_{\text {eff }}$ model in the quintessence-like dark energy scenario $\left(w_{x}>-1\right)$ from several data set combinations, where CB, CR19, CPCC, CBPCC, and CR19PCC correspond to the combinations CMB + BAO, CMB + R19, $\mathrm{CMB}+$ Pantheon $+\mathrm{CC}, \mathrm{CMB}+\mathrm{BAO}+$ Pantheon $+\mathrm{CC}$, and CMB $+\mathrm{R} 19+$ Pantheon $+\mathrm{CC}$, respectively.

\begin{tabular}{|c|c|c|c|c|c|c|}
\hline Parameters & $\mathrm{CMB}$ & $\mathrm{CB}$ & CR19 & $\mathrm{CPCC}$ & CBPCC & CR19PCC \\
\hline$\Omega_{c} h^{2}$ & $<0.113$ & $0.074_{-0.053}^{+0.046}$ & $<0.065$ & $<0.112$ & $0.077_{-0.057}^{+0.045}$ & $0.064_{-0.056}^{+0.047}$ \\
\hline$\Omega_{b} h^{2}$ & $0.02223_{-0.00044}^{+0.00044}$ & $0.02231_{-0.00040}^{+0.00041}$ & $0.02237_{-0.00037}^{+0.00040}$ & $0.02232_{-0.00037}^{+0.00038}$ & $0.02237_{-0.00036}^{+0.00037}$ & $0.02254_{-0.00036}^{+0.00037}$ \\
\hline $100 \theta_{M C}$ & $1.0448_{-0.0042}^{+0.0050}$ & $1.0439_{-0.0033}^{+0.0040}$ & $1.0475_{-0.0037}^{+0.0031}$ & $1.0439_{-0.0034}^{+0.0047}$ & $1.0437_{-0.0032}^{+0.0043}$ & $1.0445_{-0.0035}^{+0.0045}$ \\
\hline$\tau$ & $0.053_{-0.015}^{+0.016}$ & $0.055_{-0.015}^{+0.016}$ & $0.054_{-0.016}^{+0.017}$ & $0.054_{-0.015}^{+0.016}$ & $0.055_{-0.015}^{+0.016}$ & $0.056_{-0.015}^{+0.016}$ \\
\hline$n_{s}$ & $0.960_{-0.018}^{+0.017}$ & $0.963_{-0.015}^{+0.015}$ & $0.965_{-0.014}^{+0.015}$ & $0.963_{-0.014}^{+0.014}$ & $0.966_{-0.013}^{+0.014}$ & $0.973_{-0.013}^{+0.013}$ \\
\hline $\ln \left(10^{10} A_{s}\right)$ & $3.038_{-0.036}^{+0.037}$ & $3.041_{-0.035}^{+0.036}$ & $3.044_{-0.036}^{+0.037}$ & $3.042_{-0.034}^{+0.035}$ & $3.044_{-0.035}^{+0.036}$ & $3.054_{-0.034}^{+0.035}$ \\
\hline$w_{x}$ & $<-0.76$ & $<-0.81$ & $<-0.902$ & $<-0.79$ & $<-0.79$ & $<-0.823$ \\
\hline$\xi$ & $<0.27$ & $<0.24$ & $0.229_{-0.090}^{+0.069}$ & $0.13_{-0.12}^{+0.13}$ & $<0.24$ & $0.15_{-0.11}^{+0.12}$ \\
\hline$\Omega_{m 0}$ & $0.19_{-0.16}^{+0.16}$ & $0.21_{-0.12}^{+0.11}$ & $0.092_{-0.061}^{+0.082}$ & $0.21_{-0.13}^{+0.11}$ & $0.22_{-0.13}^{+0.10}$ & $0.17_{-0.11}^{+0.11}$ \\
\hline$\sigma_{8}$ & $1.6_{-1.1}^{+2.0}$ & $1.24_{-0.61}^{+0.99}$ & $2.6_{-1.5}^{+1.8}$ & $1.3_{-0.7}^{+1.3}$ & $1.2_{-0.6}^{+1.0}$ & $1.5_{-0.8}^{+1.5}$ \\
\hline$H_{0}[\mathrm{~km} / \mathrm{s} / \mathrm{Mpc}]$ & $68.1_{-7.5}^{7.3}$ & $68.1_{-2.9}^{+3.0}$ & $73.2_{-2.5}^{2.4}$ & $68.0_{-2.5}^{+2.6}$ & $68.2_{-2.1}^{+2.1}$ & $70.9_{-1.9}^{+2.0}$ \\
\hline$N_{\text {eff }}$ & $2.92_{-0.37}^{+0.38}$ & $2.97_{-0.35}^{+0.35}$ & $3.04_{-0.31}^{+0.34}$ & $3.00_{-0.31}^{+0.32}$ & $3.03_{-0.32}^{+0.32}$ & $3.23_{-0.29}^{+0.30}$ \\
\hline$S_{8}$ & $1.07_{-0.31}^{+0.51}$ & $0.97_{-0.20}^{+0.31}$ & $1.31_{-0.37}^{+0.40}$ & $0.99_{-0.22}^{+0.39}$ & $0.96_{-0.19}^{+0.32}$ & $1.04_{-0.26}^{+0.43}$ \\
\hline
\end{tabular}

The effective number of relativistic degrees of freedom $N_{\text {eff }}$ is perfectly consistent with its standard value $N_{\text {eff }}=$ $3.046[68,69]$ for all data set combinations considered here, with mean values similar to those obtained in the $w_{x}<-1$ scenario. A freely varying effective number of neutrino species $N_{\text {eff }}$ does not affect the directions of the correlations

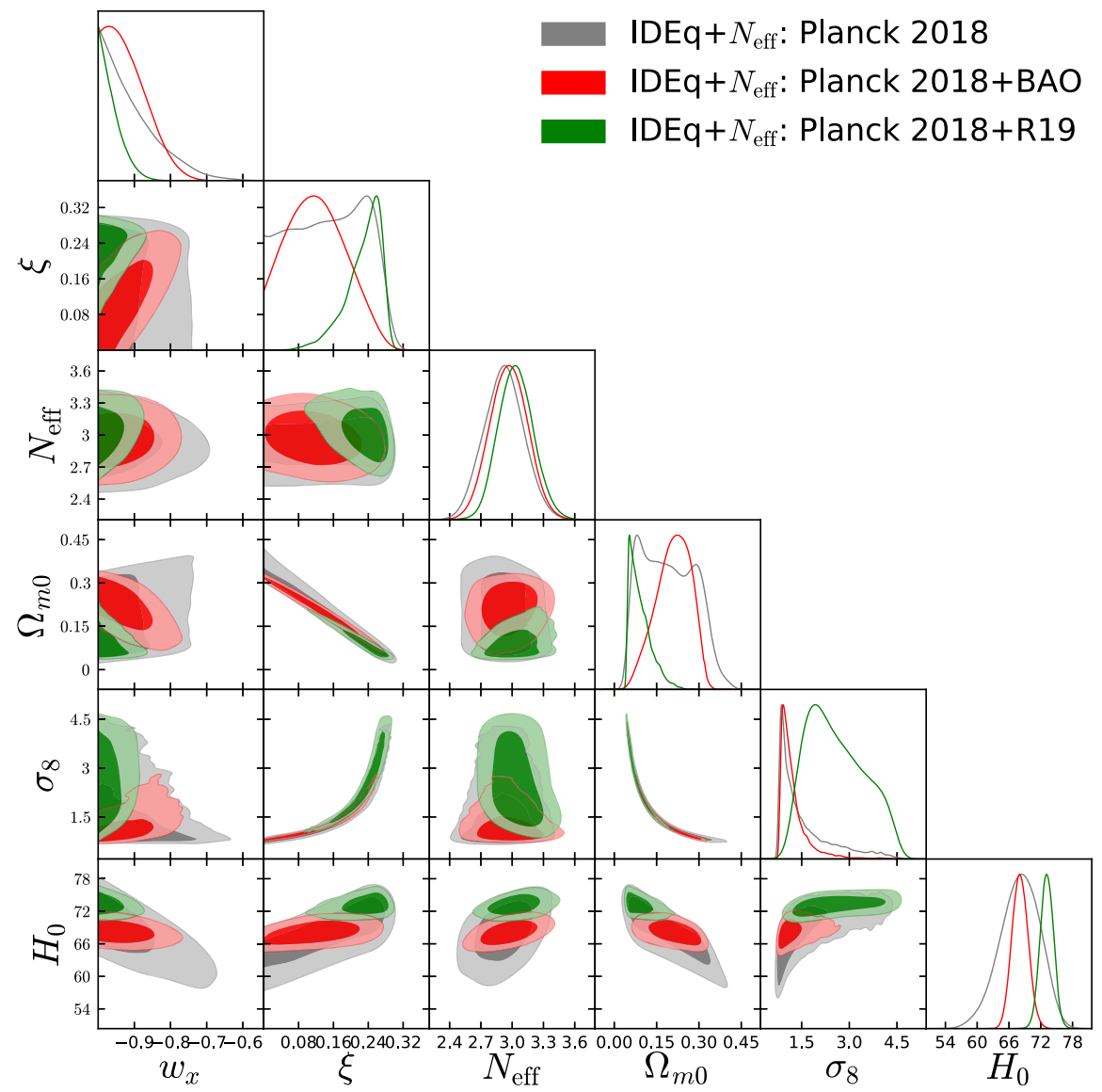

FIG. 7. $95 \%$ C.L. constraints within the IDE $+N_{\text {eff }}$ model in the quintessence-like dark energy scenario $\left(w_{x}>-1\right)$ from several data set combinations, where CB, CR19, CPCC, CBPCC, and CR19PCC correspond to the combinations CMB + BAO, CMB + R19, $\mathrm{CMB}+$ Pantheon $+\mathrm{CC}, \mathrm{CMB}+\mathrm{BAO}+$ Pantheon $+\mathrm{CC}$, and CMB + R19 + Pantheon + CC, respectively. 
TABLE IX. $95 \%$ C.L. constraints within the IDE $+M_{\nu}+N_{\text {eff }}$ model in the quintessence-like dark energy scenario $\left(w_{x}>-1\right)$ from several data set combinations, where CB, CR19, CPCC, CBPCC, and CR19PCC correspond to the combinations CMB $+\mathrm{BAO}$, $\mathrm{CMB}+\mathrm{R} 19, \mathrm{CMB}+$ Pantheon $+\mathrm{CC}, \mathrm{CMB}+\mathrm{BAO}+$ Pantheon $+\mathrm{CC}$, and CMB + R19 + Pantheon + CC, respectively.

\begin{tabular}{|c|c|c|c|c|c|c|}
\hline Parameters & $\mathrm{CMB}$ & $\mathrm{CB}$ & CR19 & СРCC & CBPCC & CR19PCC \\
\hline$\Omega_{c} h^{2}$ & $<0.113$ & $0.070_{-0.058}^{+0.049}$ & $\ll 0.061$ & $0.074_{-0.055}^{+0.046}$ & $0.077_{-0.058}^{+0.045}$ & $0.064_{-0.057}^{+0.046}$ \\
\hline$\Omega_{b} h^{2}$ & $0.02222_{-0.00045}^{+0.00046}$ & $0.02231_{-0.00039}^{+0.000040}$ & $0.02238_{-0.00036}^{+0.00039}$ & $0.02232_{-0.00038}^{+0.00040}$ & $0.02236_{-0.00035}^{+0.00036}$ & $0.02254_{-0.00036}^{+0.00036}$ \\
\hline $100 \theta_{M C}$ & $1.0448_{-0.0042}^{+0.0049}$ & $1.0442_{-0.0036}^{+0.0045}$ & $1.0476_{-0.0034}^{+0.0030}$ & $1.0439_{-0.0032}^{+0.0042}$ & $1.0437_{-0.0032}^{+0.00044}$ & $1.0444_{-0.0035}^{+0.0046}$ \\
\hline$\tau$ & $0.053_{-0.016}^{+0.016}$ & $0.054_{-0.016}^{+0.016}$ & $0.054_{-0.016}^{+0.017}$ & $0.054_{-0.015}^{+0.016}$ & $0.055_{-0.015}^{+0.016}$ & $0.056_{-0.015}^{+0.016}$ \\
\hline$n_{s}$ & $0.959_{-0.017}^{+0.017}$ & $0.962_{-0.015}^{+0.016}$ & $0.966_{-0.014}^{+0.014}$ & $0.963_{-0.015}^{+0.015}$ & $0.965_{-0.013}^{+0.014}$ & $0.973_{-0.013}^{+0.013}$ \\
\hline $\ln \left(10^{10} A_{s}\right)$ & $3.039_{-0.036}^{+0.036}$ & $3.041_{-0.036}^{+0.037}$ & $3.044_{-0.037}^{+0.037}$ & $3.043_{-0.036}^{+0.036}$ & $3.044_{-0.034}^{+0.035}$ & $3.054_{-0.034}^{+0.036}$ \\
\hline$w_{x}$ & $<-0.75$ & $<-0.80$ & $<-0.90$ & $<-0.80$ & $<-0.79$ & $<-0.818$ \\
\hline$\xi$ & $<0.28$ & $<0.26$ & $0.231_{-0.086}^{+0.068}$ & $<0.25$ & $<0.24$ & $0.15_{-0.11}^{+0.12}$ \\
\hline$\Omega_{m 0}$ & $0.19_{-0.16}^{+0.16}$ & $0.20_{-0.13}^{+0.12}$ & $0.091_{-0.056}^{+0.075}$ & $0.21_{-0.12}^{+0.11}$ & $0.22_{-0.13}^{+0.10}$ & $0.17_{-0.11}^{+0.11}$ \\
\hline$\sigma_{8}$ & $1.6_{-1.1}^{+1.9}$ & $1.3_{-0.7}^{+1.3}$ & $2.6_{-1.5}^{+1.7}$ & $1.3_{-0.6}^{+1.0}$ & $1.2_{-0.6}^{+1.0}$ & $1.5_{-0.9}^{+1.6}$ \\
\hline$H_{0}$ & $67.7_{-7.4}^{+7.3}$ & $68.1_{-2.9}^{+3.3}$ & $73.2_{-2.4}^{+2.4}$ & $68.0_{-2.6}^{+2.7}$ & $68.2_{-2.1}^{+2.2}$ & $70.8_{-1.9}^{+2.0}$ \\
\hline$M_{\nu}$ & $<0.337$ & $<0.170$ & $<0.199$ & $<0.243$ & $<0.156$ & $<0.158$ \\
\hline$N_{\text {eff }}$ & $2.92_{-0.37}^{+0.38}$ & $2.96_{-0.35}^{+0.37}$ & $3.05_{-0.32}^{+0.33}$ & $3.01_{-0.32}^{+0.33}$ & $3.02_{-0.30}^{+0.33}$ & $3.22_{-0.29}^{+0.29}$ \\
\hline$\Omega_{\nu} h^{2}$ & $<0.0035$ & $<0.0018$ & $<0.0021$ & $<0.0026$ & $<0.0017$ & $<0.0017$ \\
\hline$S_{8}$ & $1.06_{-0.30}^{+0.49}$ & $1.00_{-0.23}^{+0.39}$ & $1.32_{-0.38}^{+0.40}$ & $0.98_{-0.20}^{+0.32}$ & $0.96_{-0.20}^{+0.33}$ & $1.04_{-0.26}^{+0.43}$ \\
\hline
\end{tabular}

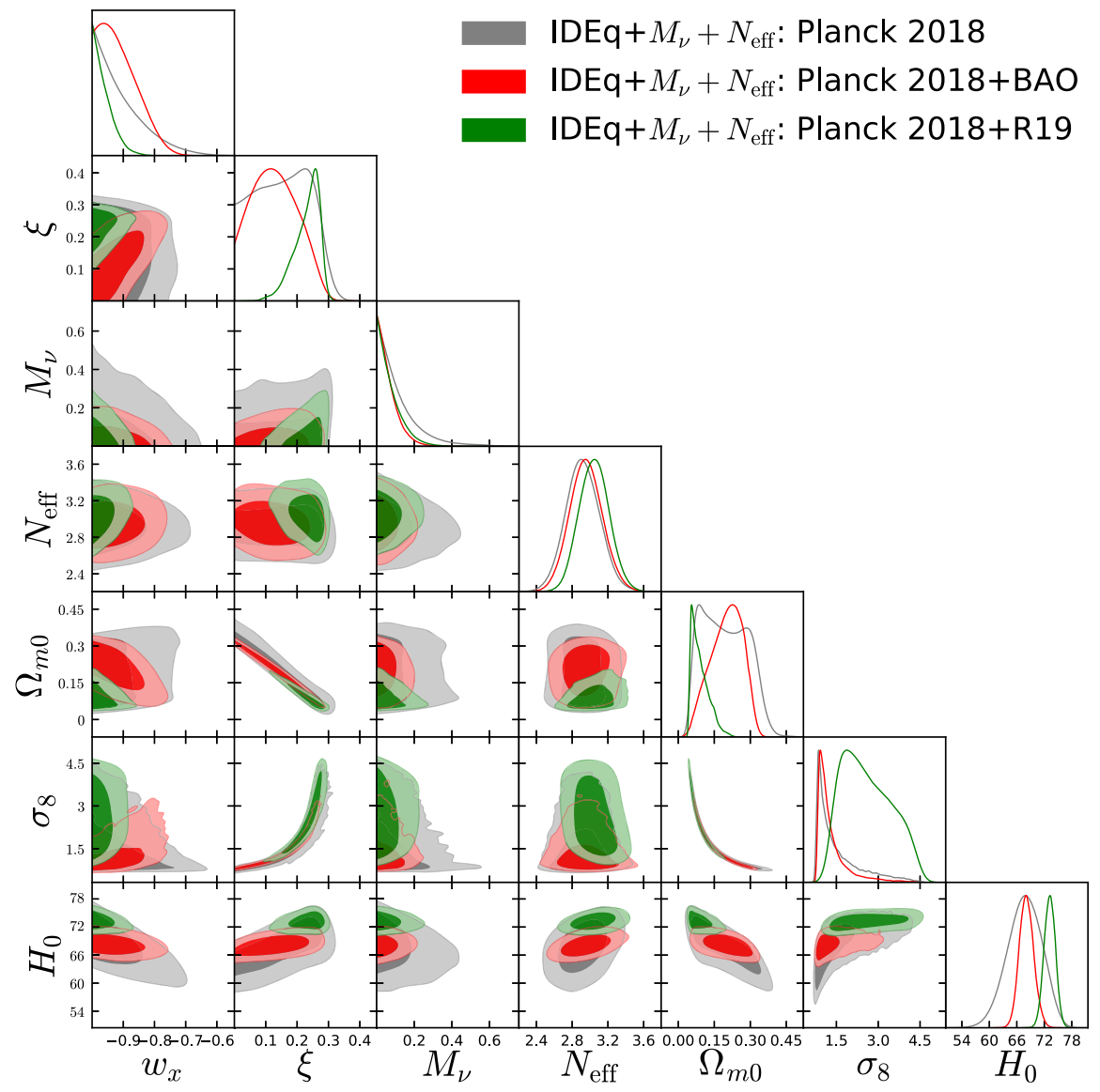

FIG. 8. $95 \%$ C.L. constraints within the IDE $+M_{\nu}+N_{\text {eff }}$ in the quintessence-like dark energy scenario $\left(w_{x}>-1\right)$ from several data set combinations, where CB, CR19, CPCC, CBPCC, and CR19PCC correspond to the combinations CMB + BAO, CMB + R19, $\mathrm{CMB}+$ Pantheon $+\mathrm{CC}, \mathrm{CMB}+\mathrm{BAO}+$ Pantheon $+\mathrm{CC}$, and CMB + R19 + Pantheon + CC, respectively. 
between the cosmological parameters, as we can see in Fig. 7, while the very strong correlation between $N_{\text {eff }}$ and the Hubble constant $H_{0}$ present within the $\Lambda$ CDM model is considerably reduced in this interacting scenario. The $N_{\text {eff }}$ values obtained for the $\mathrm{CMB}+\mathrm{R} 19, \mathrm{CMB}+$ Pantheon + $\mathrm{CC}$, and $\mathrm{CMB}+\mathrm{BAO}+$ Pantheon $+\mathrm{CC}$ data combinations are very similar and very close to 3 . However, if we consider the $\mathrm{CMB}+\mathrm{R} 19+$ Pantheon $+\mathrm{CC}$ data combination (see the last column of Table VIII), we obtain $N_{\text {eff }}=$ $3.23_{-0.29}^{+0.30}$ at $95 \%$ C.L.

\section{IDE $+M_{\nu}+N_{\text {eff }}:$ Ten parameters}

The results for this scenario are shown in Table IX and Fig. 8. In this full scenario, where both the total neutrino mass $M_{\nu}$ and the effective number of relativistic degrees of freedom $N_{\text {eff }}$ vary simultaneously, both $n_{s}$ and $H_{0}$ are shifted to lower values when compared to the previous IDE $+N_{\text {eff }}$ model shown in Table VIII.

The upper limits on the total neutrino mass $M_{\nu}$ are only mildly relaxed with respect to the IDE $+M_{\nu}$ model shown in Table III for the CMB-only case, while they are slightly stronger for the $\mathrm{CMB}+$ Pantheon $+\mathrm{CC}$ data set combination. The most stringent upper limit we have on this parameter is obtained for the data set combination $\mathrm{CMB}+$ $\mathrm{BAO}+$ Pantheon $+\mathrm{CC}$ (see the sixth column of Table IX), for which $M_{\nu}<0.156 \mathrm{eV}$ at $95 \%$ C.L.

\section{SUMMARY}

In this paper we have explored possible extensions of interacting dark energy, where the dark energy and dark matter fluids interact with each other. We have focused on the impact of such interactions on the dark radiation sector, allowing the neutrino mass and effective number of neutrino species to vary freely, both individually and simultaneously. The effect of such a dark coupling on the dark radiation sector was analyzed within two different dark energy regimes: a phantom scenario $\left(w_{x}<-1\right)$ and a quintessence scenario $\left(w_{x}>-1\right)$. We have exploited the most recent publicly available cosmological observations, which include the Planck 2018 legacy data, baryon acoustic oscillations, the most recent measurement of the Hubble constant using Cepheids as calibrators, type Ia supernovae Pantheon data, and measurements of the Hubble parameter from cosmic chronometers. We found, in general, that the constraints on the dark radiation sector physics are quite close to those found within the minimal $\Lambda \mathrm{CDM}$ cosmology. The derived bounds are almost independent of the dark energy regime (phantom versus quintessence), contrary to the case without a dark coupling $[71,72]$. We found a total neutrino mass $M_{\nu}<0.15 \mathrm{eV}$ and a number of effective relativistic degrees of freedom $N_{\text {eff }}=3.03_{-0.33}^{+0.33}$, both at $95 \%$ C.L., which are indeed not that far from those obtained within the $\Lambda$ CDM cosmology, $M_{\nu}<0.12 \mathrm{eV}$ and $N_{\text {eff }}=3.00_{-0.35}^{+0.36}$, for the same data combination. Current cosmological observations are therefore powerful enough to disentangle the physical effects associated with the different dark sector components, i.e., dark energy, dark matter, and dark radiation.

On the other hand, it might be interesting to investigate the mutual interaction scenario in the full dark sector of the Universe (namely, between dark energy, dark matter, and dark radiation) and consider different rates of interaction, and examine how such coupling can affect both the dark and neutrino sectors in light of recent cosmological observations.

\section{ACKNOWLEDGMENTS}

W. Y. acknowledges the support from the National Natural Science Foundation of China under Grants No. 11705079 and No. 11647153. E. D. V. was supported from the European Research Council in the form of a Consolidator Grant No. 681431. O. M. is supported by the Spanish Grants No. FPA2017-85985-P and No. SEV-20140398 of the MINECO, by PROMETEO/2019/083 and by the European Union Horizon 2020 research and innovation program (Grant agreements No. 690575 and 67489). S. P. has been supported by the Mathematical Research ImpactCentric Support Scheme (MATRICS), File No. MTR/2018/ 000940, given by the Science and Engineering Research Board (SERB), Govt. of India. R. C. N. would like to thank the FAPESP for financial support under the Project No. 2018/18036-5.
[1] N. Aghanim et al. (Planck Collaboration), Planck 2018 results. V. CMB power spectra and likelihoods, arXiv:1907 .12875 .

[2] N. Aghanim et al. (Planck Collaboration), Planck 2018 results. VI. Cosmological parameters, arXiv:1807 .06209

[3] N. Aghanim et al. (Planck Collaboration), Planck 2018 results. VIII. Gravitational lensing, arXiv:1807.06210.
[4] N. Suzuki et al., The hubble space telescope cluster supernova survey: V. Improving the dark energy constraints above $z>1$ and building an early-type-hosted supernova sample, Astrophys. J. 746, 85 (2012).

[5] M. Crocce et al. (DES Collaboration), Galaxy clustering, photometric redshifts and diagnosis of systematics in the DES Science Verification data, Mon. Not. R. Astron. Soc. 455, 4301 (2016). 
[6] S. Alam et al. (SDSS-III Collaboration), The eleventh and twelfth data releases of the sloan digital sky survey: Final data from SDSS-III, Astrophys. J. Suppl. Ser. 219, 12 (2015).

[7] G. Hinshaw et al. (WMAP Collaboration), Nine-year wilkinson microwave anisotropy probe (WMAP) observations: Cosmological parameter results, Astrophys. J. Suppl. Ser. 208, 19 (2013).

[8] P. A. R. Ade et al. (Planck Collaboration), Planck 2015 results. XIII. Cosmological parameters, Astron. Astrophys. 594, A13 (2016).

[9] A. P. Billyard and A. A. Coley, Interactions in scalar field cosmology, Phys. Rev. D 61, 083503 (2000).

[10] J. D. Barrow and T. Clifton, Cosmologies with energy exchange, Phys. Rev. D 73, 103520 (2006).

[11] L. Amendola, G. C. Campos, and R. Rosenfeld, Consequences of dark matter-dark energy interaction on cosmological parameters derived from SNIa data, Phys. Rev. D 75, 083506 (2007).

[12] J. H. He and B. Wang, Effects of the interaction between dark energy and dark matter on cosmological parameters, J. Cosmol. Astropart. Phys. 06 (2008) 010.

[13] J. Väliviita, E. Majerotto, and R. Maartens, Instability in interacting dark energy and dark matter fluids, J. Cosmol. Astropart. Phys. 07 (2008) 020.

[14] M. B. Gavela, D. Hernandez, L. L. Honorez, O. Mena, and S. Rigolin, Dark coupling, J. Cosmol. Astropart. Phys. 07 (2009) 034.

[15] E. Majerotto, J. Valiviita, and R. Maartens, Adiabatic initial conditions for perturbations in interacting dark energy models, Mon. Not. R. Astron. Soc. 402, 2344 (2010).

[16] M. B. Gavela, L. L. Honorez, O. Mena, and S. Rigolin, Dark coupling and gauge invariance, J. Cosmol. Astropart. Phys. 11 (2010) 044.

[17] T. Clemson, K. Koyama, G. B. Zhao, R. Maartens, and J. Valiviita, Interacting Dark Energy_constraints and degeneracies, Phys. Rev. D 85, 043007 (2012).

[18] S. Pan and S. Chakraborty, Will there be again a transition from acceleration to deceleration in course of the dark energy evolution of the universe?, Eur. Phys. J. C 73, 2575 (2013).

[19] W. Yang and L. Xu, Testing coupled dark energy with large scale structure observation, J. Cosmol. Astropart. Phys. 08 (2014) 034.

[20] W. Yang and L. Xu, Cosmological constraints on interacting dark energy with redshift-space distortion after Planck data, Phys. Rev. D 89, 083517 (2014).

[21] S. Pan, S. Bhattacharya, and S. Chakraborty, An analytic model for interacting dark energy and its observational constraints, Mon. Not. R. Astron. Soc. 452, 3038 (2015).

[22] S. Pan and G. S. Sharov, A model with interaction of dark components and recent observational data, Mon. Not. R. Astron. Soc. 472, 4736 (2017).

[23] A. Mukherjee and N. Banerjee, In search of the dark matter dark energy interaction: A kinematic approach, Classical Quantum Gravity 34, 035016 (2017).

[24] G. S. Sharov, S. Bhattacharya, S. Pan, R. C. Nunes, and S. Chakraborty, A new interacting two fluid model and its consequences, Mon. Not. R. Astron. Soc. 466, 3497 (2017).
[25] W. Yang, N. Banerjee, and S. Pan, Constraining a dark matter and dark energy interaction scenario with a dynamical equation of state, Phys. Rev. D 95, 123527 (2017).

[26] W. Yang, S. Pan, and J. D. Barrow, Large-scale stability and astronomical constraints for coupled dark-energy models, Phys. Rev. D 97, 043529 (2018).

[27] W. Yang, S. Pan, and D. F. Mota, Novel approach toward the large-scale stable interacting dark-energy models and their astronomical bounds, Phys. Rev. D 96, 123508 (2017).

[28] S. Pan, A. Mukherjee, and N. Banerjee, Astronomical bounds on a cosmological model allowing a general interaction in the dark sector, Mon. Not. R. Astron. Soc. 477, 1189 (2018).

[29] W. Yang, S. Pan, and A. Paliathanasis, Cosmological constraints on an exponential interaction in the dark sector, Mon. Not. R. Astron. Soc. 482, 1007 (2019).

[30] W. Yang, S. Pan, L. Xu, and D. F. Mota, Effects of anisotropic stress in interacting dark matter-dark energy scenarios, Mon. Not. R. Astron. Soc. 482, 1858 (2019).

[31] W. Yang, S. Pan, R. Herrera, and S. Chakraborty, Largescale (in) stability analysis of an exactly solved coupled dark-energy model, Phys. Rev. D 98, 043517 (2018).

[32] M. Martinelli, N. B. Hogg, S. Peirone, M. Bruni, and D. Wands, Constraints on the interacting vacuum-geodesic CDM scenario, Mon. Not. R. Astron. Soc. 488, 3423 (2019).

[33] A. Paliathanasis, S. Pan, and W. Yang, Dynamics of nonlinear interacting dark energy models, Int. J. Mod. Phys. D 28, 1950161 (2019).

[34] S. Pan, W. Yang, C. Singha, and E. N. Saridakis, Observational constraints on sign-changeable interaction models and alleviation of the $H_{0}$ tension, Phys. Rev. D 100, 083539 (2019).

[35] W. Yang, S. Pan, E. Di Valentino, B. Wang, and A. Wang, Forecasting interacting vacuum-energy models using gravitational waves, arXiv:1904.11980.

[36] W. Yang, S. Vagnozzi, E. Di Valentino, R. C. Nunes, S. Pan, and D.F. Mota, Listening to the sound of dark sector interactions with gravitational wave standard sirens, J. Cosmol. Astropart. Phys. 07 (2019) 037.

[37] G. Papagiannopoulos, P. Tsiapi, S. Basilakos, and A. Paliathanasis, Dynamics and cosmological evolution in $\Lambda$-varying cosmology, Eur. Phys. J. C 80, 55 (2020).

[38] S. Kumar and R. C. Nunes, Echo of interactions in the dark sector, Phys. Rev. D 96, 103511 (2017).

[39] E. Di Valentino, A. Melchiorri, and O. Mena, Can interacting dark energy solve the $H_{0}$ tension?, Phys. Rev. D 96, 043503 (2017).

[40] W. Yang, S. Pan, E. Di Valentino, R. C. Nunes, S. Vagnozzi, and D.F. Mota, Tale of stable interacting dark energy, observational signatures, and the $H_{0}$ tension, J. Cosmol. Astropart. Phys. 09 (2018) 019.

[41] W. Yang, A. Mukherjee, E. Di Valentino, and S. Pan, Interacting dark energy with time varying equation of state and the $H_{0}$ tension, Phys. Rev. D 98, 123527 (2018).

[42] S. Kumar and R. C. Nunes, Probing the interaction between dark matter and dark energy in the presence of massive neutrinos, Phys. Rev. D 94, 123511 (2016).

[43] S. Kumar and R. C. Nunes, Observational constraints on dark matter - dark energy scattering cross section, Eur. Phys. J. C 77, 734 (2017). 
[44] S. Kumar, R. C. Nunes, and S. K. Yadav, Dark sector interaction: A remedy of the tensions between $\mathrm{CMB}$ and LSS data, Eur. Phys. J. C 79, 576 (2019).

[45] W. Yang, S. Pan, R. C. Nunes, and D. F. Mota, Dark calling Dark: Interaction in the dark sector in presence of neutrino properties after Planck CMB final release, arXiv:1910 .08821

[46] J. Gleyzes, D. Langlois, M. Mancarella, and F. Vernizzi, Effective theory of interacting dark energy, J. Cosmol. Astropart. Phys. 08 (2015) 054.

[47] S. Pan, G. S. Sharov, and W. Yang, Field theoretic interpretations of interacting dark energy scenarios and recent observations, arXiv:2001.03120.

[48] S. Pan, J. de Haro, W. Yang, and J. Amorós, Understanding the phenomenology of interacting dark energy scenarios and their theoretical bounds, arXiv:2001.09885.

[49] F. Beutler, C. Blake, M. Colless, D. H. Jones, L. StaveleySmith, L. Campbell, Q. Parker, W. Saunders, and F. Watson, The $6 \mathrm{dF}$ galaxy survey: Baryon acoustic oscillations and the local hubble constant, Mon. Not. R. Astron. Soc. 416, 3017 (2011).

[50] A. J. Ross, L. Samushia, C. Howlett, W. J. Percival, A. Burden, and M. Manera, The clustering of the SDSS DR7 main Galaxy sample C I. A 4 per cent distance measure at $z=0.15$, Mon. Not. R. Astron. Soc. 449, 835 (2015).

[51] S. Alam et al. (BOSS Collaboration), The clustering of galaxies in the completed SDSS-III Baryon Oscillation Spectroscopic Survey: cosmological analysis of the DR12 galaxy sample, Mon. Not. R. Astron. Soc. 470, 2617 (2017).

[52] A. G. Riess, S. Casertano, W. Yuan, L. M. Macri, and D. Scolnic, Large magellanic cloud cepheid standards provide a $1 \%$ foundation for the determination of the hubble constant and stronger evidence for physics beyond

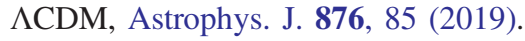

[53] D. M. Scolnic et al., The complete light-curve sample of spectroscopically confirmed SNe Ia from Pan-STARRS1 and cosmological constraints from the combined pantheon sample, Astrophys. J. 859, 101 (2018).

[54] M. Moresco, L. Pozzetti, A. Cimatti, R. Jimenez, C. Maraston, L. Verde, D. Thomas, A. Citro, R. Tojeiro, and D. Wilkinson, A $6 \%$ measurement of the Hubble parameter at $z \sim 0.45$ : direct evidence of the epoch of cosmic reacceleration, J. Cosmol. Astropart. Phys. 05 (2016) 014.

[55] A. Lewis and S. Bridle, Cosmological parameters from CMB and other data: A Monte Carlo approach, Phys. Rev. D 66, 103511 (2002).

[56] A. Lewis, A. Challinor, and A. Lasenby, Efficient computation of CMB anisotropies in closed FRW models, Astrophys. J. 538, 473 (2000).

[57] A. Gelman and D. Rubin, Inference from iterative simulation using multiple sequences, Stat. Sci. 7, 457 (1992).
[58] E. Di Valentino, A. Melchiorri, O. Mena, and S. Vagnozzi, Non-minimal dark sector physics and cosmological tensions, Phys. Rev. D 101, 063502 (2020).

[59] T. M. C. Abbott et al. (DES Collaboration), Dark energy survey year 1 results: Cosmological constraints from galaxy clustering and weak lensing, Phys. Rev. D 98, 043526 (2018).

[60] M. A. Troxel et al. (DES Collaboration), Dark energy survey year 1 results: Cosmological constraints from cosmic shear, Phys. Rev. D 98, 043528 (2018).

[61] H. Hildebrandt et al., KiDS-450: Cosmological parameter constraints from tomographic weak gravitational lensing, Mon. Not. R. Astron. Soc. 465, 1454 (2017).

[62] K. Kuijken et al., Gravitational lensing analysis of the kilo degree survey, Mon. Not. R. Astron. Soc. 454, 3500 (2015).

[63] I. F. Conti, R. Herbonnet, H. Hoekstra, J. Merten, L. Miller, and M. Viola, Calibration of weak-lensing shear in the Kilo-Degree Survey, Mon. Not. R. Astron. Soc. 467, 1627 (2017).

[64] C. Heymans et al., CFHTLenS: The Canada-France-Hawaii telescope lensing survey, Mon. Not. R. Astron. Soc. 427, 146 (2012).

[65] T. Erben et al., CFHTLenS: The Canada-FranceHawaii telescope lensing survey-Imaging data and catalogue products, Mon. Not. R. Astron. Soc. 433, 2545 (2013).

[66] S. Joudaki et al., CFHTLenS revisited: Assessing concordance with Planck including astrophysical systematics, Mon. Not. R. Astron. Soc. 465, 2033 (2017).

[67] G. La Vacca, S. A. Bonometto, and L. P. L. Colombo, Higher neutrino mass allowed if DM and DE are coupled, New Astron. 14, 435 (2009).

[68] G. Mangano, G. Miele, S. Pastor, T. Pinto, O. Pisanti, and P. D. Serpico, Relic neutrino decoupling including flavor oscillations, Nucl. Phys. B729, 221 (2005).

[69] P. F. de Salas and S. Pastor, Relic neutrino decoupling with flavour oscillations revisited, J. Cosmol. Astropart. Phys. 07 (2016) 051.

[70] E. Di Valentino, A. Melchiorri, O. Mena, and S. Vagnozzi, Interacting dark energy after the latest Planck, DES, and $H_{0}$ measurements: An excellent solution to the $H_{0}$ and cosmic shear tensions, arXiv:1908.04281.

[71] W. Yang, R. C. Nunes, S. Pan, and D. F. Mota, Effects of neutrino mass hierarchies on dynamical dark energy models, Phys. Rev. D 95, 103522 (2017).

[72] S. Vagnozzi, S. Dhawan, M. Gerbino, K. Freese, A. Goobar, and O. Mena, Constraints on the sum of the neutrino masses in dynamical dark energy models with $w(z) \geq-1$ are tighter than those obtained in $\Lambda$ CDM, Phys. Rev. D 98, 083501 (2018). 